\title{
Structure-function relationship of $\mathrm{H} 2 \mathrm{~A}-\mathrm{H} 2 \mathrm{~B}$ specific plant histone chaperones
}

\author{
Ashish Kumar $^{1,2} \cdot$ Dileep Vasudevan $^{1}$ (D) \\ Received: 21 August 2019 / Revised: 15 October 2019 / Accepted: 28 October 2019 / Published online: 9 November 2019 \\ (C) Cell Stress Society International 2019
}

\begin{abstract}
Studies on chromatin structure and function have gained a revived popularity. Histone chaperones are significant players in chromatin organization. They play a significant role in vital nuclear functions like transcription, DNA replication, DNA repair, DNA recombination, and epigenetic regulation, primarily by aiding processes such as histone shuttling and nucleosome assembly/disassembly. Like the other eukaryotes, plants also have a highly orchestrated and dynamic chromatin organization. Plants seem to have more isoforms within the same family of histone chaperones, as compared with other organisms. As some of these are specific to plants, they must have evolved to perform functions unique to plants. However, it appears that only little effort has gone into understanding the structural features of plant histone chaperones and their structure-function relationships. Studies on plant histone chaperones are essential for understanding their role in plant chromatin organization and how plants respond during stress conditions. This review is on the structural and functional aspects of plant histone chaperone families, specifically those which bind to $\mathrm{H} 2 \mathrm{~A}-\mathrm{H} 2 \mathrm{~B}$, viz nucleosome assembly protein (NAP), nucleoplasmin (NPM), and facilitates chromatin transcription (FACT). Here, we also present comparative analyses of these plant histone chaperones with available histone chaperone structures. The review hopes to incite interest among researchers to pursue further research in the area of plant chromatin and the associated histone chaperones.
\end{abstract}

Keywords Histone chaperones $\cdot \mathrm{H} 2 \mathrm{~A}-\mathrm{H} 2 \mathrm{~B} \cdot \mathrm{NAP} 1 \cdot \mathrm{NRP} 1 \cdot \mathrm{FACT} \cdot$ Nucleoplasmin

\section{Introduction}

The genetic material of all the eukaryotic organisms including yeast, plants, and animals exist as compact chromosomes, residing in the cellular nuclei, and these in interphase and metaphase of the cell cycle are called chromatin. More specifically, chromatin consists of DNA and small molecular weight, positively charged histone proteins in association with a diverse group of non-histone chromatin proteins like high mobility group (HMG) proteins (Spiker 1985). A better understanding of chromatin organization came about when it became evident that nucleosomes are the smallest units which provide the first level of compaction to nuclear DNA (Luger

Dileep Vasudevan

dileep@ils.res.in

Institute of Life Sciences, Bhubaneswar, Odisha 751023, India

2 Manipal Academy of Higher Education,

Manipal, Karnataka 576104, India et al. 1997). The electrostatic interaction between DNA and histone octamer is a critical factor that holds the whole nucleosome core particle in a complex organization. This electrostatic interaction breaks during several crucial nuclear processes that require nucleosome disassembly, like DNA repair, DNA recombination, and transcription. Once these processes get completed, the nucleosomes should reassemble. During nucleosome disassembly, histones come out from their complex octameric form as $\mathrm{H} 2 \mathrm{~A}-\mathrm{H} 2 \mathrm{~B}$ and need to get protected from the inappropriate non-nucleosomal charge interactions. A specific class of nuclear proteins called histone chaperones shields the charge-based interactions of the histones. Histone chaperones aid nucleosome assembly as well. Apart from these functions, they also take part in the trafficking of histones inside and outside the nucleus (Avvakumov et al. 2011).

Histone chaperones are specific to the histones they bind and rely on particular motifs for interacting with the histones. Moreover, some of the histone chaperones are capable of distinguishing between different histone types, as well as between canonical and variant histones. Within a nucleosome, $(\mathrm{H} 3-\mathrm{H} 4)_{2}$ or histone tetramer is located internally, and as such 
the exchange of H2A-H2B dimer occurs more quickly; mostly during replication-independent assembly or disassembly of the nucleosome, a process aided by the histone chaperones. Among these, nucleosome assembly protein (NAP) (Park and Luger 2006a, 2006b; Park and Luger 2008), facilitates chromatin transcription (FACT) (Orphanides et al. 1999), nucleoplasmin (NPM) (Laskey et al. 1978), acidic nuclear phosphoprotein 32e (Anp32e) (Obri et al. 2014), and histone acetyltransferase 1-interacting factor 1 (Hifl) (Zhang et al. 2016) are known to be H2A-H2B specific chaperones. Similarly, anti-silencing function-1 (ASF1) (Le et al. 1997), chromatin assembly factor-1 (CAF1) (Smith and Stillman 1989), nuclear autoantigenic sperm protein (NASP) (Avvakumov et al. 2011), etc. are H3-H4 specific chaperones. Histone chaperones such as vacuolar protein sortingassociated protein 72 homolog (VPS72) (Wu et al. 2005) and chaperone for Htz1/H2A-H2B dimer (Chz1) (Hansen et al. 2009; Zhou et al. 2008) are specific for H2A.Z-H2B dimers. Death domain-associated protein (Daxx) (Drane et al. 2010; Elsasser 2013; Elsasser et al. 2012) and histone cell cycle regulation defective homolog A (HIRA) (Elsaesser and Allis 2010; Elsasser 2013) are chaperones known to be specific for H3.3-H4 (Avvakumov et al. 2011). Of these, NAP, CAF, FACT, ASF1, HIRA, and NASP family proteins have been reported from plants as well (Maksimov et al. 2016; Tripathi et al. 2015; Yoon et al. 1995; Zhou et al. 2016). Since H2A-H2B gets evicted first during nucleosome disassembly and is deposited last during the assembly process, its deposition and eviction could provide dynamic nature to the chromatin. Thus, histone H2A-H2B chaperones are critical players. The structure of different yeast and human histone chaperones have been solved to understand their role in regulating chromatin dynamics. More recently, the structures of histone chaperones in complex with histones, as well as various transcription factors, have also started coming out. These structural studies provide a much deeper insight into the function of these enigmatic proteins. Having a structure-based understanding of chromatin rearrangements could be quite significant for dealing with the disease conditions in animals and stress responses in plants.

Plants have more isoforms within the same family of histone chaperones, as compared with other organisms. As some of these are specific to plants, they must have evolved to perform functions unique to plants. However, the structural and functional studies of these histone chaperones in plants have not gained much attention. Plants undergo several biotic and abiotic stress conditions, and histone chaperones form one of the crucial factors for their survival. Moreover, no reviews discuss the structural aspects of plant histone chaperones and showcase the importance of structural studies on plant histone chaperones and their complexes. So, we thought it would be useful to review the structural details of H2A-H2B histone chaperones and their complexes with histone dimers as well as other interacting partners to understand their functional attributes. In this review, we cover primarily about the known structural and functional features of plant $\mathrm{H} 2 \mathrm{~A}-\mathrm{H} 2 \mathrm{~B}$ chaperones belonging to the families such as NAP, nucleoplasmins, and FACT and try to bring out the importance of carrying out structural studies on plant histone chaperones.

\section{Nucleosome assembly proteins}

Nucleosome assembly proteins (NAP) belong to a large histone chaperone family having the conserved $\alpha / \beta$-earmuff motif (Park and Luger 2006b). NAP1, initially obtained from Xenopus laevis eggs and identified as a protein that facilitates the in vitro assembly of nucleosomes, is conserved extensively across different species (Laskey et al. 1978). NAP family proteins have been reported from a wide range of organisms, such as yeast (Ishimi and Kikuchi 1991), Plasmodium (Gill et al. 2009), Drosophila (Ito et al. 1996), Xenopus (Steer et al. 2003), human (Li et al. 2016), Caenorhabditis elegans (Sarkar et al. 2019), soya bean (Yoon et al. 1995), rice (Dong et al. 2003), tobacco (Dong et al. 2003), and Arabidopsis (Liu et al. 2009). The representative members of NAP family include Vps75 in yeast (Berndsen et al. 2008; Tang et al. 2008), hsNAPIL and xINAPIL in human and Xenopus laevis, respectively, SET $\alpha$ and SET $\beta$ in humans and metazoans, and NAP1 related protein 1 (NRP1) and NAP1 related protein 2 (NRP2) in A. thaliana (Zhu et al. 2006). To date, NAP1- and NAP1related protein structures are available only from a few organisms, as listed in Table 1.

The NAP1 protein in plants was first isolated from cDNA clones of soya bean (Glycine max). G. $\max$ NAP1 shows $32 \%$ and $38 \%$ sequence similarity with human and $S$. cerevisiae NAP1, respectively (Yoon et al. 1995). NAP1 proteins have also been identified from other plants like A. thaliana (AtNAP1), Oryza sativa (OsNAP1), Zea mays (ZmNAP1), and Nicotiana tabacum (NtNAP1) (Dong et al. 2003). Arabidopsis and rice, both contain four different isoforms of NAP1 namely, NAP1;1, NAP1;2, NAP1;3 and NAP1;4 with two additional members from the same family called NAP1-related proteins (NRPs). The NRPs have conserved domains similar to NAP1, but differs phylogenetically and in structure from NAP1 (Kumar et al. 2019; Liu et al. 2009; Zhu et al. 2006). When we compared AtNAP1 isoforms with OsNAP1 isoforms, we found that all these NAP1 proteins contain an extended coiled-coil region of around 55 residues, predicted nuclear export and import sequences and a long Glu/Asp containing disordered tract of about 85 residues at the $\mathrm{C}$-terminus. The presence of these disordered regions appears to be a characteristic feature of this family of proteins. Figure 1 a shows a comparison of the domain organization in ScNAP1 and AtNAP1. 
Table 1 Structures of NAP family proteins and their complexes

\begin{tabular}{|c|c|c|c|c|c|}
\hline $\begin{array}{l}\text { Name of the } \\
\text { chaperone }\end{array}$ & $\begin{array}{l}\text { Source } \\
\text { organism }\end{array}$ & $\begin{array}{l}\text { PDB } \\
\text { code }\end{array}$ & $\begin{array}{l}\text { Residue } \\
\text { range }\end{array}$ & Resolution & Primary citation \\
\hline ScNAP1 & S. cerevisiae & $2 \mathrm{AYU}$ & $1-417$ & $3.0 \AA$ & $\begin{array}{l}\text { (Park and Luger } \\
\text { 2006b) }\end{array}$ \\
\hline ScNAP1 & S. cerevisiae & $2 \mathrm{Z} 2 \mathrm{R}$ & $74-365$ & $3.2 \AA$ & (Park et al. 2008) \\
\hline PfNAPL & P. falciparum & $3 \mathrm{FS} 3$ & $1-359$ & $2.3 \AA$ & (Gill et al. 2009) \\
\hline PfNAPL & & $3 \mathrm{GYW}$ & $1-249$ & $2.4 \AA$ & (Yogavel et al. 2009) \\
\hline PfNAPL (SAD) & & $3 \mathrm{GYV}$ & $1-249$ & $3.0 \AA$ & (Yogavel et al. 2009) \\
\hline PkNAPl & P. knowlesi & 3HFD & $1-276$ & $2.8 \AA$ & (Unpublished) \\
\hline PfNAPS & P. falciparum & $5 \times 7 \mathrm{~V}$ & $29-221$ & $2.8 \AA$ & (Gill et al. 2010) \\
\hline CeNAP1 & C. elegans & $6 \mathrm{~N} 2 \mathrm{G}$ & $10-293$ & $3.0 \AA$ & (Sarkar et al. 2019) \\
\hline HsTAF-I $\beta$ & H. sapiens & $2 \mathrm{E} 50$ & $1-225$ & $2.3 \AA$ & (Muto et al. 2007) \\
\hline AtNRP1 & A. thaliana & 5DAY & $19-225$ & $2.3 \AA$ & (Zhu et al. 2017) \\
\hline AtNRP2 & A. thaliana & & $14-223$ & $3.4 \AA$ & (Kumar et al. 2019) \\
\hline ScVPS75 & S. cerevisiae & 2ZD7 & $1-264$ & $1.85 \AA$ & (Park et al. 2008) \\
\hline ScVPS75 & S. cerevisiae & 3DM7 & $1-234$ & $2.0 \AA$ & (Tang et al. 2008) \\
\hline ScVPS75 & & $3 \mathrm{C} 9 \mathrm{D}$ & $1-259$ & $2.0 \AA$ & (Berndsen et al. 2008) \\
\hline ScVPS75 (SeMet) & & $3 \mathrm{C} 9 \mathrm{~B}$ & $1-259$ & $2.4 \AA$ & (Berndsen et al. 2008) \\
\hline ScVPS75 tetramer & S. cerevisiae & $5 \mathrm{AGC}$ & $1-264$ & $4.0 \AA$ & (Hammond et al. 2016) \\
\hline ScVPS75:RTT109 & S. cerevisiae & 3Q35 & $\begin{array}{l}9240 \\
\text { (VPS75) } \\
1-438 \\
\text { (RTT109) }\end{array}$ & $3.3 \AA$ & (Tang et al. 2011) \\
\hline $\begin{array}{l}\text { ScVPS75:RTT109:H3 } \\
\text { peptide }\end{array}$ & S. cerevisiae & 3Q33 & $\begin{array}{l}9-240 \\
\text { (VPS75) } \\
1-438 \\
\text { (RTT109) } \\
11-25(\mathrm{H} 3)\end{array}$ & $2.8 \AA$ & (Tang et al. 2011) \\
\hline ScVPS75:RTT109 & S. cerevisiae & $\begin{array}{l}\text { 3Q66 } \\
\text { 3Q68* }\end{array}$ & $\begin{array}{c}1-264 \\
\text { (VPS75) } \\
1-442 \\
\text { (RTT109) } \\
1-264 \\
\text { (VPS75) } \\
1-442 \\
\text { (RTT109) }\end{array}$ & $\begin{array}{l}2.7 \AA \\
2.7 \AA\end{array}$ & $\begin{array}{l}\text { (Su et al. 2011) } \\
(\mathrm{Su} \text { et al. 2011) }\end{array}$ \\
\hline ScNAP1:H2A-H2B & $\begin{array}{l}\text { S. cerevisiae } \\
\text { X. laevis } \\
\quad \text { (Histones) }\end{array}$ & $5 \mathrm{G} 2 \mathrm{E}$ & $\begin{array}{c}82-391 \\
\text { (NAP1) } \\
16-122 \\
(\mathrm{H} 2 \mathrm{~A}) \\
34-103 \\
(\mathrm{H} 2 \mathrm{~B})\end{array}$ & $6.7 \AA$ & $\begin{array}{l}\text { (Aguilar-Gurrieri et al. } \\
\text { 2016) }\end{array}$ \\
\hline
\end{tabular}

*Different space group
ScNAP1 is $48 \mathrm{kDa}$ in size, forms a homodimer and interacts with $\mathrm{H} 2 \mathrm{~A}-\mathrm{H} 2 \mathrm{~B}, \mathrm{H} 3-\mathrm{H} 4$, and $\mathrm{H} 1$, and is capable of mediating nucleosome assembly in vitro (Park and Luger 2006a; Park and Luger 2006b; Zlatanova et al. 2007). However, AtNAP $1 ; 1$ is a $43 \mathrm{kDa}$ protein, $39.75 \%$ similar to ScNAP1. Sequence alignment of AtNAP1;1, OsNAP1;1 and ScNAP1 shows a high degree of sequence conservation among the three, indicating similar secondary structural organization (Fig. 1b). To further probe the possible structural differences between AtNAP1 and ScNAP1, we generated a homology model of AtNAP1;1 (residues 30-310) monomer using Phyre 2 modeling server (Kelley et al. 2015). The quality of the model was assessed by using ProQ2 quality assessment tool (Ray et al. 2012). The Ramachandran plot analysis was 


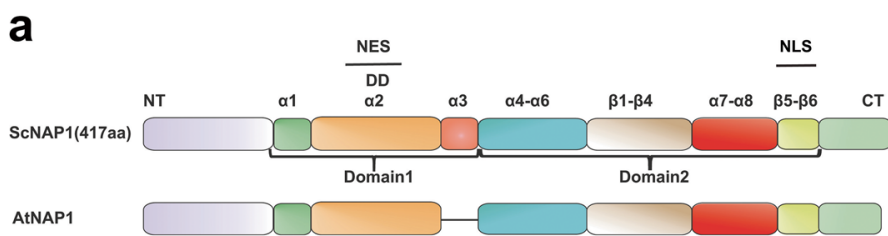

b

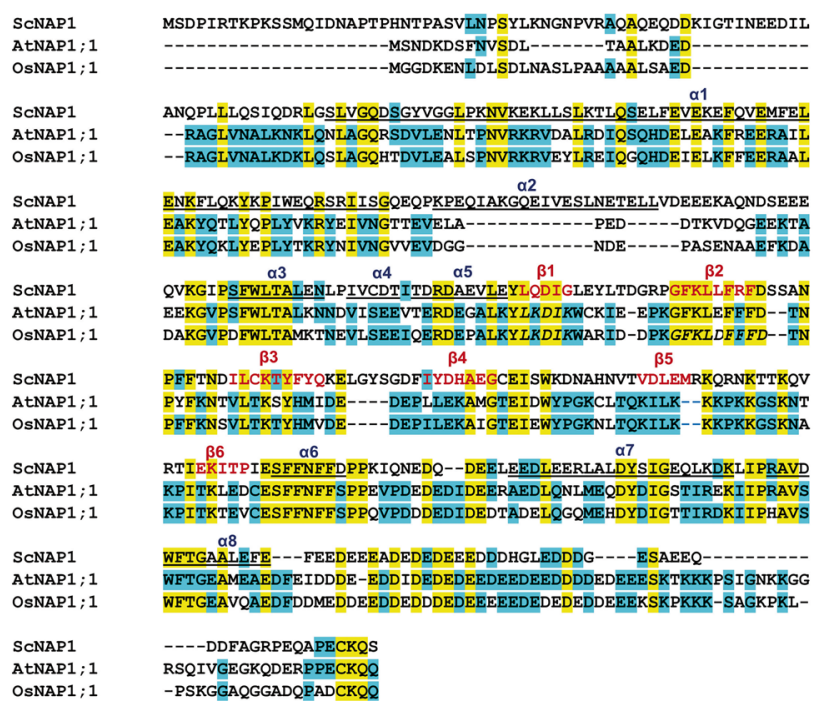

Fig. 1 Domain organization, sequence alignment, and structure alignments of ScNAP1 and AtNAP1. a The figure shows the similarities and differences in the domain organization of $S$. cerevisiae and $A$. thaliana NAP1 proteins. The N-terminal disordered tail (NT), the dimerization domain (DD), the disordered C-terminal tail with Asp/Glu residues (CT), the nuclear export sequence (NES), and the nuclear localization sequence (NLS) are labeled. AtNAP1 has four different isoforms (not shown in this figure), with a similar domain organization, but differing in length, ranging from 317 to 379 residues. b Sequence alignment of AtNAP1;1, OsNAP1;1, and ScNAP1. Highlighted in yellow are the

done using Phyre 2 investigator (Kelley et al. 2015) and RAMPAGE (Lovell et al. 2003), both.

The r.m.s. deviation upon the alignment of the modeled AtNAP1 structure with the crystal structure of ScNAP1 was 0.57 for $171 \mathrm{C} \alpha$ atoms; however for CeNAP1, it was 1.095 for $167 \mathrm{C} \alpha$ atoms. The overall domain organization of AtNAP1 is similar to ScNAP1 (Fig. 1c) with some significant differences within the domain I. The long dimerization helix $\alpha 2$ of AtNAP1 shows an offset of $20.2^{\circ}$ as compared with that of ScNAP1 (Fig. 1c). The offset in $\alpha 2$ causes another offset of $-70.8^{\circ}$ in the helix $\alpha-1$ of AtNAP1, which brings $\alpha 1$ and $\mathrm{N}$-terminal region of the protein closer to $\alpha 2$ as well as the $\beta$-sheet regions of domain II. The twisting of $\alpha 1$ seems to be essential for stabilization by interaction with acidic areas in domain II (Fig. 1d). This kind of arrangement may provide more acidic surface area, which aids histone interaction. The nuclear localization sequence (residues 223-228) is present in $\beta 5$ of the $\beta$-hairpin region formed by $\beta 5$ and $\beta 6$,
C
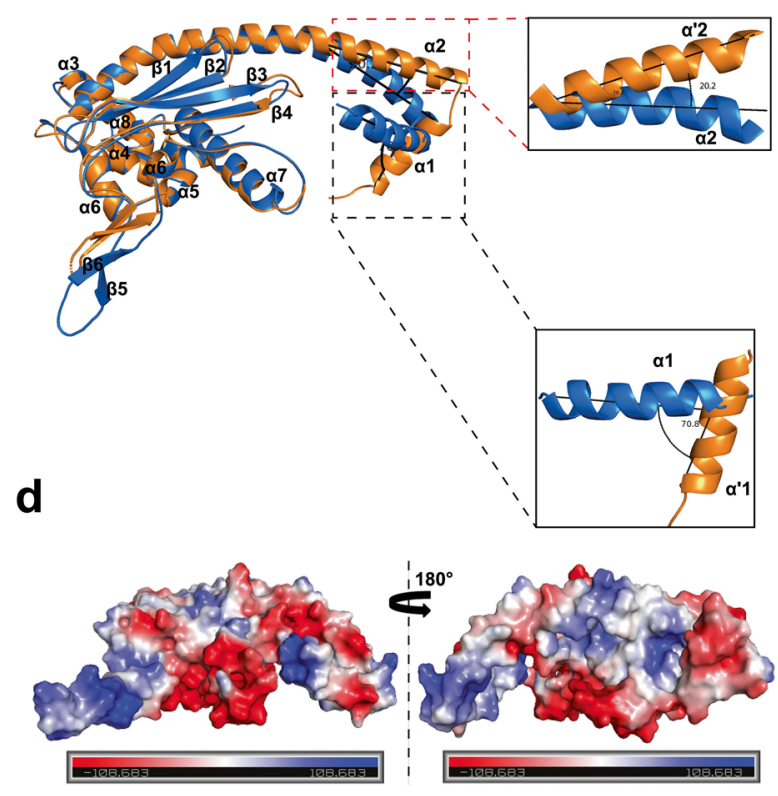

residues conserved in all three species; highlighted in cyan are residues conserved in at least two species. For the ScNAP1 sequence, underlined are the residues forming $\alpha$-helices, and shown in red are the residues forming $\beta$-sheets. ClustalW multiple sequence alignment tool aided the alignment. c Structure alignment of modeled AtNAP1 (blue) with ScNAP1 (orange). The r.m.s.d deviation for AtNAP1 structure superimposed with ScNAP1 is 0.57 for $171 \mathrm{C} \alpha$ atoms. The zoomed boxes show the offsets in the structure. $\mathbf{d}$ Surface charge distribution of AtNAP1 monomer model. Phyre2 server was used for the homology modeling

and at the $\mathrm{N}$-terminus of $\alpha 2$ is the nuclear export sequence (residues 47-62), similar to that of ScNAP1. The amino acid sequence in $\beta 5$ of ScNAP1 is considerably different from the corresponding stretch of AtNAP1;1 and OsNAP1;1 (Fig. 1b) and thus the plane of $\beta$-hairpin seems slightly different in AtNAP1. The presence of both NLS and NES in NAP proteins and their homologs hints towards their nucleo-cytoplasmic shuttling and possible roles in both cytoplasm and nucleus.

Interestingly, the NAP1-related proteins, NRP1, and NRP2 do not contain any NLS, but they possess NES (Tripathi et al. 2015). The NRP proteins are mostly nuclear-localized (Zhu et al. 2006) and hence the absence of NLS in NRP1 and NRP2 is still a puzzle, as in how they get transported into the nucleus. The lack of NLS in NRP proteins and their presence in the nucleus suggests they have a critical nuclear function. NAP proteins could move in and out of the nucleus, and thus, it could have more of histone transport functions. However, since NRP 
proteins are nuclear-localized, they could be more involved in the regulation of transcription. Thus, studies on NRP proteins and their interaction with various transcription factors could be something exciting to address, especially during the stress conditions. The presence of some post-translational modifications may be utilized for its transport, similar to Drosophila NAP1 (Ito et al. 1996) or maybe, association with histones aid their transport to the nucleus. The presence of NES suggests a continuous shuttling also as a possibility. A difference in the nucleocytoplasmic shuttling of NAP1 proteins is found in different plant species, suggesting that these processes could be dependent on sequence and post-translational modifications of the proteins (Zhou et al. 2015).

The only structures known from the plant NAP family are those of A. thaliana NRP1 and NRP2. Although the NRP1 and NRP2 are about $87 \%$ identical in protein sequence, a relatively higher r.m.s.d value $(3.24 \AA$ for 323 $\mathrm{C} \alpha$ atoms) was seen for their structure superposition (Kumar et al. 2019). A $26^{\circ}$ offset in dimerization helix of AtNRP2 leads to the overall difference in the structure of NRP2 dimer as compared with NRP1 dimer. Also, there is a possibility of forming a higher-order oligomer for both AtNRP1 and AtNRP2, as could be deduced from the crystal packing of AtNRP2 to form tetramers (Kumar et al. 2019). The differences in the overall rate of oligomerization of these proteins might account for the differences in histone carrying capacity of the two (Kumar et al. 2019). The interaction studies with histone dimers, and both AtNRP1, and AtNRP2 further shows that AtNRP2 possesses a higher binding affinity for histone dimers than AtNRP1 and thus, AtNRP2 could be more efficient as a histone chaperone. Further comparison of both AtNRP1 and AtNRP 2 structures with that of HsSET/TAFI- $\beta$ has provided some clues about the histone-binding moieties in these two proteins. Certain mutational studies on HsTAF-I $\beta$ called $\mathrm{N}, \mathrm{O}$, and $\mathrm{P}$ mutations have revealed the possible site of histone binding. Based on structural comparison, six residues of this site in HsTAF-I $\beta$ appear to be conserved and present in both AtNRP1 and AtNRP2 structure as well, which corresponds to residues T191, T194, D195, D202, E203, and E206 respectively of HsTAF-I 3 (PDB id, 2E50) (Muto et al. 2007; Zhu et al. 2017).

AtNRP1 is crucial in regulating several defense-related genes during biotic stress conditions (Barna et al. 2018). A comparative study of AtNRP1 and AtNRP2 functions during biotic stress requires more attention and the structural studies on these proteins with various transcription factors could help us understand their role during stress conditions. Also, looking at the changes in expression of these histone chaperones during stress conditions could improve our understanding of these proteins in plant survival.

\section{NAP1-histone complex structures}

Hydrogen-Deuterium exchange (H/DX) studies have shown that $\mathrm{H} 2 \mathrm{~A}-\mathrm{H} 2 \mathrm{~B}$ dimer remains in a partially unfolded conformation in the absence of ScNAP1 and that the presence of NAP1 reduces this conformational sampling by forming $\mathrm{H}-$ bonds with the histone fold regions of these histones (D'Arcy et al. 2013). This histone fold interaction is quite similar to what is observed in a nucleosome octamer structure. It was also shown that the NAP1 helical underside interacts directly with H2A-H2B dimer near to its L2 loop. Moreover, NAP1 dimer was found to bind with two copies of H2A-H2B (D'Arcy et al. 2013). However, the crystal structure of ScNAP1 in complex with H2A-H2B revealed that the NAP1 dimer binds to a single H2A-H2B by providing an acidic binding surface (Aguilar-Gurrieri et al. 2016), thereby contradicting the previous report. The structural studies illustrate that the core domain (75-365) of NAP1 is sufficient for histone binding, as well as for nucleosome assembly. The dimer of ScNAP1 was present in an antiparallel fashion where its concave face provides the binding sites for $\mathrm{H} 2 \mathrm{~A}-\mathrm{H} 2 \mathrm{~B}$ dimer (Fig. 2c). Figure $2 \mathrm{~d}$ shows the details of the interacting residues of NAP1 and the histones. NAP1 mimics the structure and the electrostatic interaction with $\mathrm{H} 2 \mathrm{~A}-\mathrm{H} 2 \mathrm{~B}$ and wraps around it in a fashion similar to DNA, engaging two of the three DNA binding surfaces of the H2A-H2B dimer, with the majority of the contacts towards H2A. The H/DX study (D'Arcy et al. 2013) made use of the full-length ScNAP1 protein, and the crystallographic work (Aguilar-Gurrieri et al. 2016) used a truncated version of the protein. The discrepancy in the results possibly came about due to differences in the length of ScNAP1 used in the two works.

In plants, AtNRPs could bind to two copies of histone H2A-H2B dimers (Kumar et al. 2019). However, no such information is available for AtNAP1 proteins. Further structural studies on plant NAP1 and NAP1 related proteins (NRPs) with histone dimers are required to address the actual functional differences in the function of these two groups of proteins. The studies on post-translational modifications on NAPs and NRPs would help in exploring the interactions in a more physiologically relevant manner.

\section{Nucleoplasmins}

Historically, a particular category of proteins, found in very high concentrations in the soluble phase of the nucleus and not bound to chromatin was termed nucleoplasmins (Earnshaw et al. 1980). It was initially identified and purified from the eggs of $X$. laevis and was found capable of catalyzing nucleosome assembly in vitro, using purified core histones and DNA (Chen et al. 1994; Earnshaw et al. 1980; Laskey et al. 1978). Later, nucleoplasmins were defined as histone 

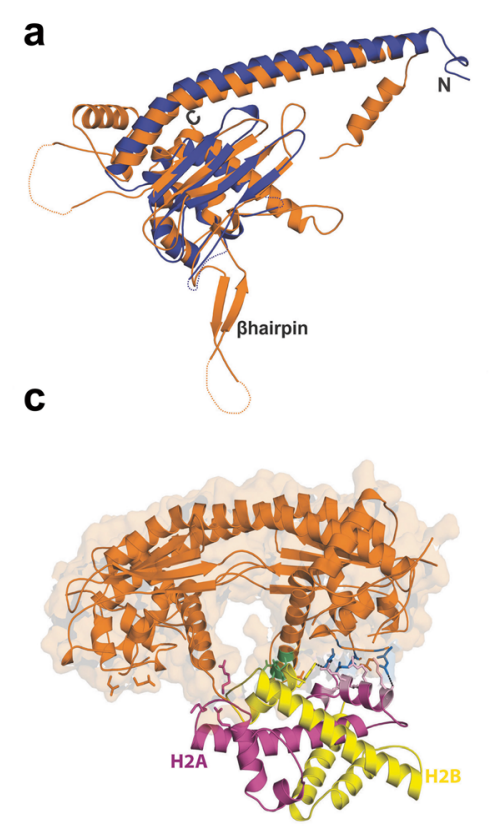

Fig. 2 Structure of NAP family proteins. a The structure superposition of ScNAP1 (PDB id, 2AYU) with AtNRP1 (PDB id, 5DAY). The structures align with an r.m.s.d. of $2.63 \AA$. AtNRP1 is in blue and ScNAP1 in orange. The dotted lines represent the flexible loop regions missing in the crystal structures. b The structure superposition of AtNRP1 (PDB id, 5DAY) and AtNRP2 (PDB id, 6JQV) with human SET/TAF-I 3 (PDB id, 2E50). The structures align with an r.m.s.d. of $2.37 \AA$. AtNRP1 is in blue, AtNRP2 in yellow, and SET/TAF-I $\beta$ in cyan. Most of the secondary structural features align well in the three structures. The dotted lines represent the flexible loop regions missing in the crystal structures. c

chaperones because of their specific interaction with histones. They are involved in shielding the charge of histones, as well as storing and transporting histones during nucleosome assembly. Although the discovery of nucleoplasmins (NPMs) was from $X$. laevis eggs, they exist in somatic cell nuclei as well, but with a slightly higher molecular weight than oocyte NPM (Cotten et al. 1986). The primary function of NPM appears to be in maintaining an ionic environment conducive to nucleosome assembly. Later it was also found to be significant in sperm decondensation in Xenopus eggs during fertilization, in gene transcription activity, enhancement of ribosomal DNA (rDNA) transcription, proper centrosome duplication, and as an adaptor, tethering the centromeres to nucleolus (Chen et al. 1994; Earnshaw et al. 1980; Padeken et al. 2013; Philpott et al. 1991). Nucleoplasmins are highly acidic, thermophilic, and karyophilic proteins. They oligomerize into a doughnut-shaped pentamer, capable of binding all four canonical histones in vitro and H2A-H2B specifically in vivo (Dutta et al. 2001; Earnshaw et al. 1980; Laskey et al. 1978; Philpott et al. 1991; Sugawa et al. 1985). NPMs have been reported to be present in vertebrates, birds, and mammals (Sugawa et al. 1985), and based on phylogenetic studies they are believed to be present in arthropods, fungi, and plants as well (Edlich-Muth et al. 2015). However, the earliest evidence

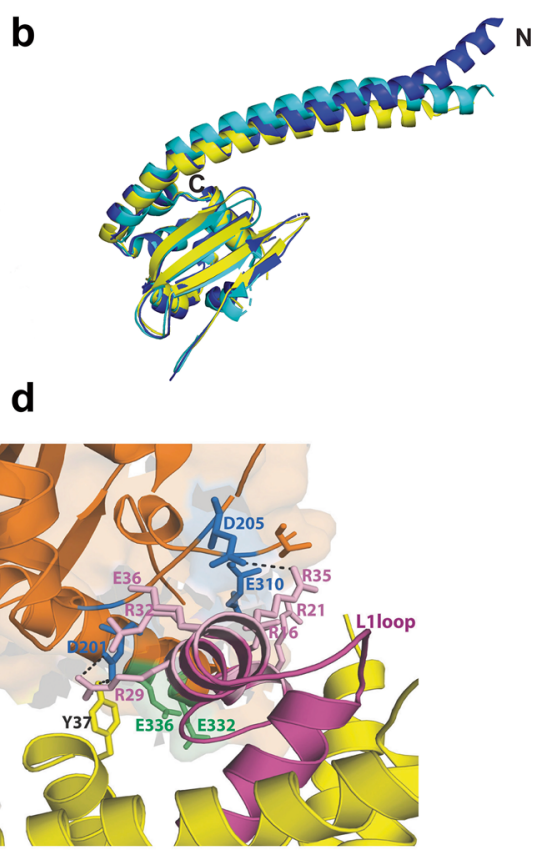

The structure of ScNAP1 in complex with histone H2A-H2B (PDB id, $5 \mathrm{G} 2 \mathrm{E}$ ). The dimeric structure of ScNAP1 reveals the mode of interaction with $\mathrm{H} 2 \mathrm{~A}-\mathrm{H} 2 \mathrm{~B}$. d A zoomed view of the interaction between ScNAP1 and the histones. Histone H2A is in violet, and H2B is in yellow. The residues forming the histone-binding region 1 (HBR1) of ScNAP1 are represented as blue sticks (D201, D205, and E310). The two significant residues of histone-binding region 2 (HBR2) are shown in green (E332 and E336). The L1 loop of H2A, which packs the HBR1 of ScNAP1, is also labeled.

for the presence of nucleoplasmins in plants was reported as the presence of a thermostable NPM-like protein in wheat embryos, capable of converting histones and DNAs into nucleosome (Laskey et al. 1978).

The architecture of nucleoplasmin protein was understood from the studies where the cDNA coding for NPM was cloned from $X$. laevis oocyte expression library (Burglin and De Robertis 1987). The analysis of protein sequence showed the $\mathrm{N}$-terminal core region that is extensively hydrophobic to be responsible for pentamer formation. However, the C-terminus was found to have an uncommon and exceptionally hydrophilic stretch of twelve glutamic acid residues, which is now known to be a characteristic feature of most of the other known histone chaperones. Also, the last 50 residues are rich in Lys and Ala residues and contain a short stretch of sequence, homologous to histone $\mathrm{H} 1$, which was predicted to be involved in nucleosome interaction (Burglin and De Robertis 1987). In addition to the nucleosome interaction sites at the $\mathrm{C}$-terminal tail region, it also contains a nuclear localization sequence. The first structure of the nucleoplasmin core revealed it to be a stable pentamer (Dutta et al. 2001). Typical nucleoplasmin consists of a stable $\mathrm{N}$-terminal core domain and a disordered C-terminal region (Fig. 3a). The protein contains one acidic tract made up of Asp and Glu residues in the 
a

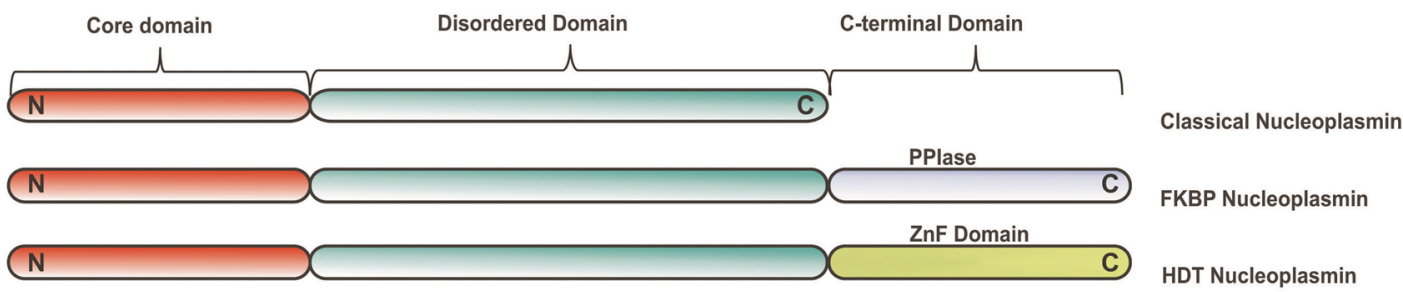

b

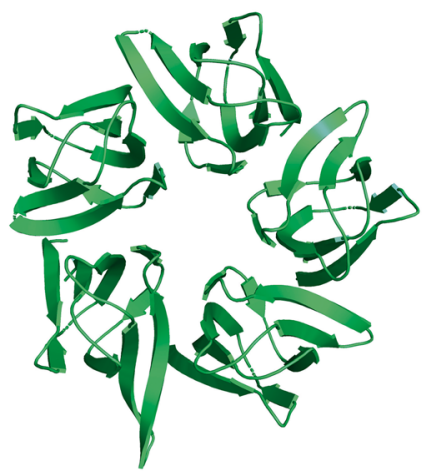

C

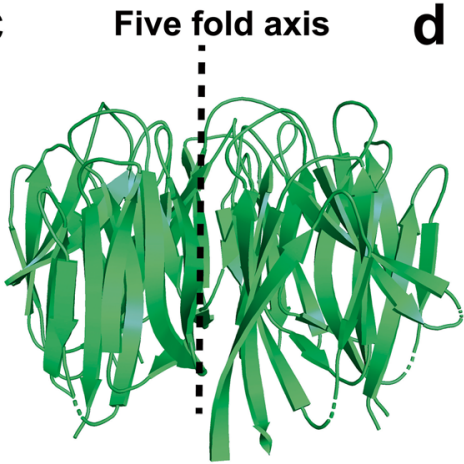

HDT Nucleoplasmin

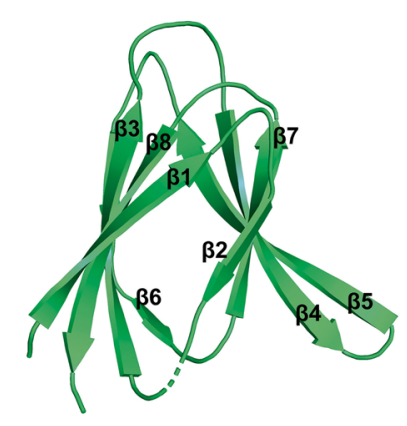

Fig. 3 Domain organization and structure of nucleoplasmins. a The domain organization of the three classes of nucleoplasmins. The domains are labeled. The N-terminal core domain forms a pentamer in all nucleoplasmins. b, c The pentameric structure of the N-terminal

$\mathrm{N}$-terminal core and two to three additional acidic stretches in the $\mathrm{C}$-terminus and a nuclear localization sequence in between the acidic tracts.

Several nucleoplasmin family proteins are present throughout the animal kingdom. Based on the sequence features, nucleoplasmins got classified into four groups; NPM1, NPM2, NPM3, and the invertebrate NPM proteins (Frehlick et al. 2006). With reports on nucleoplasmins from plants, now they have been broadly classified into three major classes based on their structural similarity and the presence of additional domains at $\mathrm{C}$-terminus. The three types are: (1) nucleoplasmins with an $\mathrm{N}$-terminal nucleoplasmin domain and a disordered C-terminus, (2) FKBP-nucleoplasmins with an N-terminal nucleoplasmin domain, a disordered middle domain and a C-terminal FK506-binding or PPIase domain and, (3) HD-tuins with N-terminal nucleoplasmin domain and a zinc finger motif at the C-terminus (Fig. 3a) (Edlich-Muth et al. 2015). Only recent literature have started reporting the presence of nucleoplasmins in fungi and plants (Edlich-Muth et al. 2015), and it needs further experimental validation to prove their role as nucleoplasmins.

The crystal structure of the $\mathrm{N}$-terminal domain of nucleoplasmins shows all of them to form a homopentamer, where each monomer is made up of eight $\beta$-strands arranged in antiparallel fashion giving it a jellyroll topology and a triangular cross-section (Fig. 3b-d). The $\beta$-strands within each monomer show a parallel alignment to the 5 -fold axis and the domain of $X$. laevis nucleoplasmin (PDB id, $1 \mathrm{~K} 5 \mathrm{~J}$ ) in two different orientations. The five-fold axis is also shown in c. d The structure of an NPM monomer. The image shows the typical triangular cross-section and the arrangement of $\beta$-sheets in an NPM monomer

strands $\beta 4$ and $\beta 5$ are involved in a $\beta$-hairpin formation, which is quite crucial for the pentamer formation and its stability (Dutta et al. 2001). There are a total of seven distinct nucleoplasmin crystal structures (Table 2) - two structures each from Drosophila (DmNLP \& DmFKBP39), Xenopus (XINPM \& X1NO38 NPM) and human (HsNPM1 \& HsNPM2) nucleoplasmins, and one from mouse (MmNPM1). The Xenopus nucleoplasmin is also known to form a decamer with a 522 symmetry within the crystal, via two conserved motifs, GSGP motif (G107-P110), and AKDE motif (A56-E59). The crystal structure reveals that the pentamer-pentamer interface does not contain any solventexcluded face, and hence, decamer formation may not be very stable in solution (Dutta et al. 2001). Also, recently, it has been shown that the decamer formation does not happen for native nucleoplasmin isolated from $X$. laevis egg; however, it occurs mostly for recombinant proteins (Franco et al. 2019). The decamer forming ability is believed to be very crucial for nucleoplasmin function. The studies on human nucleoplasmin 2 (NPM2) have shown that NPM2 exists as a decamer when it binds to both $\mathrm{H} 2 \mathrm{~A}-\mathrm{H} 2 \mathrm{~B}$ dimer and $\mathrm{H} 3-\mathrm{H} 4$ tetramer, at the same time. However, NPM2 stays as a pentamer when it binds only to H2A-H2B dimer (Ramos et al. 2010). Thus, a dynamic equilibrium may also be possible between pentameric and decameric forms to facilitate the binding of different histones. The crystal structure of human NPM2 shows the characteristic 
Table 2 Structures of nucleoplasmin family proteins and their complexes

\begin{tabular}{|c|c|c|c|c|c|}
\hline Name & Source organism & $\begin{array}{l}\mathrm{PDB} / \mathrm{EMD} \\
\text { code }\end{array}$ & $\begin{array}{l}\text { Residue } \\
\text { range }\end{array}$ & Resolution & Primary citation \\
\hline Nucleoplasmin & X. laevis & $\begin{array}{l}1 \mathrm{~K} 5 \mathrm{~J} \\
2 \mathrm{VTX}\end{array}$ & $\begin{array}{l}1-124 \\
1-120\end{array}$ & $\begin{array}{l}2.3 \AA \\
2.5 \AA\end{array}$ & $\begin{array}{l}\text { (Dutta et al. } \\
\text { 2001), } \\
\text { (Taneva et al. } \\
\text { 2008) }\end{array}$ \\
\hline NO38 nucleoplasmin & X. laevis & $\begin{array}{l}1 \mathrm{XE} 0 \\
1 \mathrm{XB} 9\end{array}$ & $\begin{array}{l}1-114 \\
1-114\end{array}$ & $\begin{array}{l}1.7 \AA \\
1.9 \AA\end{array}$ & $\begin{array}{l}\text { (Namboodiri } \\
\text { et al. 2004) } \\
\text { (Namboodiri } \\
\text { et al. 2004) }\end{array}$ \\
\hline Nucleoplasmin 2 & D. melanogaster & $1 N L Q$ & $1-108$ & $1.5 \AA$ & $\begin{array}{l}\text { (Namboodiri } \\
\text { et al. 2003) }\end{array}$ \\
\hline FKBP39 nucleoplasmin & D. melanogaster & 4CA9 & $1-98$ & NMR & $\begin{array}{r}\text { (Edlich-Muth } \\
\text { et al. 2015) }\end{array}$ \\
\hline Nucleoplasmin 1 & H. sapiens & $5 \mathrm{EHD}$ & $1-116$ & $2.5 \AA$ & (Unpublished) \\
\hline Nucleoplasmin 2 & H. sapiens & $3 \mathrm{~T} 30$ & $1-110$ & $1.9 \AA$ & $\begin{array}{l}\text { (Platonova et al. } \\
\text { 2011) }\end{array}$ \\
\hline Nucleoplasmin 1 & M. musculus & $4 \mathrm{~N} 8 \mathrm{M}$ & $1-133$ & $1.8 \AA$ & $\begin{array}{l}\text { (Mitrea et al. } \\
\text { 2014) }\end{array}$ \\
\hline $\begin{array}{c}\text { Nucleoplasmin }+ \\
\text { H2A-H2B }\end{array}$ & $\begin{array}{l}\text { X. laevis } \\
\text { (Nucleoplasmi- } \\
\text { n) } \\
\text { G. gallus } \\
\text { (Histones) }\end{array}$ & $\begin{array}{l}2 \mathrm{XQL} \\
\text { EMD-1777 }\end{array}$ & $1-200$ & $19.5 \AA$ & $\begin{array}{l}\text { (Ramos et al. } \\
\text { 2010) }\end{array}$ \\
\hline $\begin{array}{l}\text { Nucleoplasmin }+ \\
\text { histone octamer }\end{array}$ & $\begin{array}{l}\text { X. laevis } \\
\text { (Nucleoplasmi- } \\
\text { n) } \\
\text { G. gallus } \\
\text { (Histones) }\end{array}$ & EMD-0323 & $1-200$ & $14.7 \AA$ & $\begin{array}{l}\text { (Franco et al. } \\
\text { 2019) }\end{array}$ \\
\hline
\end{tabular}

pentamer structure except for the absence of A1 tract in the core pentamer region, and the structure suggests that it may require the acidic stretches in the $\mathrm{C}$-terminal region for its interaction with histones (Platonova et al. 2011).

Although cross-linking and low-resolution electron microscopic studies of Xenopus egg-isolated full-length nucleoplasmins have shown their existence as a pentamer (Earnshaw et al. 1980), no crystal structures are available for the pentameric full-length nucleoplasmins due to the presence of long intrinsically disordered stretches in the region $\mathrm{C}$ terminus to the core domain. Work on the full-length DmFKBP39, through multi-angle light scattering (MALS) and small-angle X-ray scattering (SAXS), suggested that it exists as a stable tetramer in solution (Kozlowska et al. 2017). However, a report on $X$. laevis NPM have shown the full-length protein to be a pentamer with the C-terminal arms extending from each monomer and with a slightly open conformation (Warren et al. 2017). It has been shown that the tail domain could regulate the accessibility of its second acidic tract for histone binding via specific electrostatic interactions with its basic C-terminus and NLS region (Warren et al. 2017). Furthermore, a recent cryo-electron microscopic structure of the Xenopus egg nucleoplasmin in complex with the histone octamer shows that indeed the full-length nucleoplasmin exists as a pentamer and the post-translational modifications on it increase the negative charge density as well as promotes the exposure of the acidic poly-glutamate tract (Franco et al. 2019). The structure reveals two nucleoplasmin pentamers to be associating with one whole histone octamer, wherein, the tail of the chaperone could slide, making the complex heterogeneous, thereby making high-resolution data collection difficult (Franco et al. 2019).

The sequence alignment of the N-terminal region of the plant FK506-binding proteins (FKBPs) and the Class II histone deacetylases (HDTs) shows significant similarity with nucleoplasmins, including the nucleoplasmin domain of D. melanogaster FKBP39 (Bourque et al. 2016; EdlichMuth et al. 2015). Also, when we further analyzed the fulllength sequences of HDTs, we found that two of them (HDT1 and HDT3) have zinc finger motif at the C-terminal end, within the disordered stretch and the other two (HDT2 and HDT4) have only the disordered C-terminal stretches and no zinc finger motifs, similar to the classical nucleoplasmins. When we further looked at the distribution of acidic stretches in the full-length HDTs, we found the presence of three acidic tracts and a bipartite nuclear localization sequence at the $\mathrm{C}$-terminal end similar to known nucleoplasmins. 
Like other nucleoplasmins, HDTs also seem to contain several phosphorylation sites, in the stretch C-terminal to the core domain. The secondary structure prediction of these HDTs and alignment with XINPM and DmFKBP39 NPM domain shows them to possibly have a nucleoplasmin-like fold with eight $\beta$-strands in the $\mathrm{N}$-terminal region and thus these might also form pentameric structures (Fig. 4).

To further investigate, we compared the $\mathrm{N}$-terminal domain sequences of all four HDTs and FKBP-nucleoplasmins from A. thaliana, along with the nucleoplasmin domains of DmNLP, DmFKBP39, and XINPM. The sequences aligned well with significant similarity, though the identity appeared quite low between the sequences. The position of the acidic tract inside the core domain in FKBP43 and FKBP53 sequences is different from that of others. Among HDTs, only HDT2 appears to have a proper acidic tract A1, similar to XINPM and DmNLP. In the case of HDT1, it is a short, two-residue A1 tract, and HDT3 and HDT4 do not have any A1 tract in the N-terminal core domain. As such, it appears that the significant histone-binding regions of these proteins may be present in their C-terminal ends and not within the predicted $\mathrm{N}$-terminal nucleoplasmin core. The HDT and FKBP proteins are known to interact with histone H3-H4 tetramer as well and could directly interact with chromatin (Bourque et al. 2016). All these evidence strongly suggest that HDTs are members of nucleoplasmin family in plants; specifically, HDT2 and HDT4 could be classical nucleoplasmins and HDT1 and HDT3 could be divergent forms of nucleoplasmins with C-terminal zinc finger motifs.
The structural and functional characterization of these proteins may provide further significant information regarding their role as nucleoplasmins in plants. Since these proteins in higher eukaryotes are of prime importance in histone storage, especially in the oocyte, it could be interesting to look at their role in plant embryos and meristem. Also, the structural studies on FKBP-nucleoplasmins and HDTs could give more clues on their role as prolyl isomerases and histone deacetylases respectively.

\section{Nucleoplasmin-histone complex structures}

To date, no crystal structures are available for any nucleoplasmin-histone complexes; however, electron microscopy and SAXS studies show that a nucleoplasmin pentamer could bind to five molecules of histone dimer, with one dimer per NPM monomer (Ramos et al. 2010; Warren et al. 2017). The degree of hyperphosphorylation decides the actual amount of histones binding to NPM, and the binding occurs favorably on the distal face of the chaperone (Warren et al. 2017). Also, the glutamate residues (E127-E131) of Xenopus NPM interact directly with histone H2A-H2B, and thus the glutamylation at these residues could increase the affinity of histone dimers with the NPM (Warren et al. 2017). It has also been shown that the core domain and $\mathrm{A} 2$ acidic tract both are significant for the chaperoning function and deposition of histones over NPM (Warren et al. 2017). In contrast to the previous electron microscopic studies done with NPM coreH2A-H2B complex (Ramos et al. 2010), the SAXS study

$\begin{array}{llll}\beta 1 & \beta 2 & \beta 3 & \beta 4\end{array}$

XINPM
DmNLP
DmFKBP39
AtFKBP53
AtFKBP43
AtHDT4
AtHDT3
AtHDT1
AtHDT2

XINPM

DmNLP

DmFKBP39

AtFKBP53

AtFKBP43

AtHDT4

AtHDT3

AtHDT1

AtHDT2

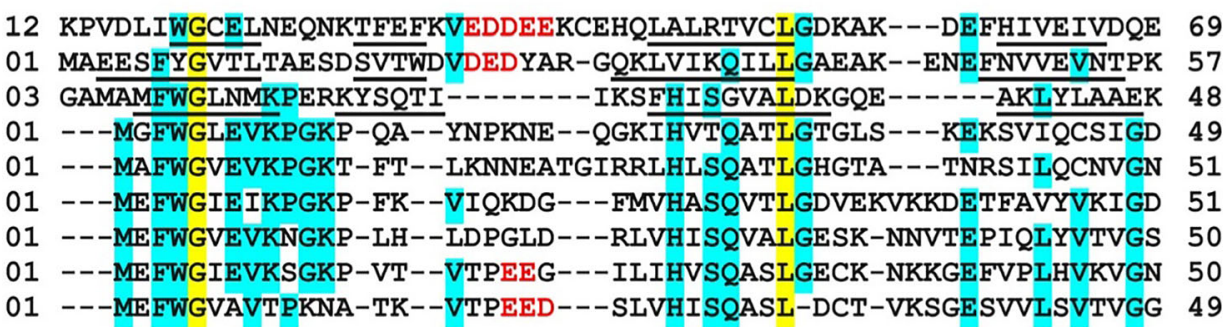

$\beta 5$

B6

$\beta 7$

$\beta 8$

70 EGAEKSVPIATLKPSILPMATMVGIEIDPP-VTFRIKAGSGPLYISGOHVAM-

58 DS--VOIPIAVLKAGETRAVNPDVEFYESK-VTFKLIKGSGPVYIHGHNIKDDV---

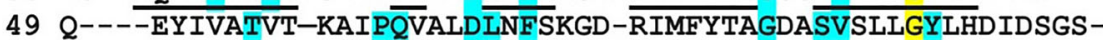
$50 \mathrm{KA}---$ PIALCSLLPNKIECCPLNLEFDDDDE PVEFTVTGD RSIHLSGFLEYYQD---

52 KS---PLLLCVLTPDKVDSCQLNLEFEETDE-VIFSVIGPRSVHLTGYFLGRS----

52 DE--NGFMIGNLS-QKFPQFSIDLYLGHEFE--ISHNST-SSVYLIGYRTFDAFD--

51 D----KLLIGTLSHEKFPOLSTEIVLERNFA--LSHTWKNGSVFFSGYKVDASDPE-

51 Q----NLVLGTLSTENIPQLFCDLVFDKEFE--LSHTWGKGSVYFVGYKTPNIEPQ50 A----KLVIGTLSQDKFPQISFDLVFDKEFE--LSHSGTKANVHFIGYKSPNIEQDD

120
110
96
100
100
100
100
100
100

120

100

100

100

100
Fig. 4 The sequence alignment of putative plant nucleoplasmins with known nucleoplasmins. The sequences of the $\mathrm{N}$-terminal core domains of HDTs and FKBP-nucleoplasmins from $A$. thaliana aligned with that of $X$. laevis nucleoplasmin, D. melanogaster nucleoplasmin-like protein, and D. melanogaster FKBP39 nucleoplasmin domain. The residues conserved across all the sequences are highlighted in yellow, residues conserved in at least five sequences are highlighted in cyan and the residues forming the acidic tract are shown in red. The $\beta$-sheet regions of known structures are labeled and underlined. The sequence alignment was done using the multiple sequence alignment tool ClustalW 
has shown that the histone dimer binds to the lateral face of the pentamer where the acidic A1 tract is present, and this mode of interaction avoids the clashes between the adjacent dimers as well (Warren et al. 2017). It appears a more plausible explanation for histone binding. Future structural studies on nucleoplasmins in complex with histones should ideally be carried out with the full-length proteins or at least a more extended stretch including the longest acidic tract (A2 tract). A recent low resolution, cryo-EM structure of $X$. laevis nucleoplasmin 2 in complex with histone octamer has shown two nucleoplasmin pentamers to bind the two opposite faces of a histone octamer core (Franco et al. 2019). The structure agrees with the previous report regarding the interaction between the A2 tract of nucleoplasmin with the N-terminal region of $\mathrm{H} 2 \mathrm{~A}$ and the $\mathrm{C}$-terminal region of $\mathrm{H} 2 \mathrm{~B}$ (Warren et al. 2017). However, this structure does not support a previous model of the human and Xenopus nucleoplasmin 2 in complex with histone octamer, where it has been proposed that the lateral surfaces of a preformed nucleoplasmin decamer possibly binds with five copies of histone octamers (Platonova et al. 2011). We believe an integrative structural biology approach for the full-length nucleoplasmins with bound histone oligomers would give a detailed understanding of the actual mode of binding and chaperone activity of these histone chaperones.

\section{Facilitates chromatin transcription}

Facilitates chromatin transcription (FACT) discovered as a factor facilitating chromatin transcription by RNA PolII, is a heterodimeric protein complex, later identified as a histone chaperone (Bondarenko et al. 2015; Hsieh et al. 2013; Orphanides et al. 1999; Reinberg and Sims 3rd 2006). A heterodimer of FACT is made up of two subunits in yeasts, plants, and animals. The first subunit is called Suppressor of Ty16 (SPT16), also known as Cdc68 and the other subunit is called structure specific recognition protein 1 (SSRP1) in humans and plants, and PolII binding protein 3 (Pob3) in yeast (Bondarenko et al. 2015; Duroux et al. 2004; Hondele et al. 2013; Oliveira et al. 2014; Tsunaka et al. 2016; Winkler and Luger 2011; Yang et al. 2016). The existence of FACT complex in plants was first identified in maize as SSRP1 protein (Rottgers et al. 2000) and then both SPT16 and SSRP1 in A. thaliana by chromatin immunoprecipitation experiments (Duroux et al. 2004). It has been reported from the rice plant as well (Tripathi et al. 2015). The mutant plant which has reduced level of expression of SPT16 or SSRP1 shows defects in vegetative and reproductive development and reduced expression of anthocyanin biosynthesis genes under light stress conditions, although they are phenotypically similar (Lolas et al. 2010; Pfab et al. 2018a; Pfab et al. 2018b).

In Arabidopsis thaliana, SPT16 is about $120 \mathrm{kDa}$ in size, and SSRP1 is approximately $71 \mathrm{kDa}$ in size. However, human
SPT16 and SSRP1 are around $116 \mathrm{kDa}$ and $78 \mathrm{kDa}$ in size, respectively. The full domain organization of yeast, human, and Arabidopsis FACT complex is depicted in Fig. 5. The Arabidopsis SPT16 and SSRP1 show $35.5 \%$ and $31 \%$ sequence identity with human SPT16 and SSRP1, respectively. Among all the organisms, SPT16 is a highly conserved subunit and consists of an N-terminal domain (NTD), a dimerization domain (DD), a middle domain (MD), and an intrinsically disordered C-terminal domain (CTD) (Fig. 5).

Structural studies have focused primarily on the various domains of SPT16 from S. cerevisiae. Structures are also available for SPT16 from human, drosophila, plant, and the fungus, Chaetomium thermophilum. A list of all the known domain structures for SPT16 from various sources and their histone complex structures, along with PDB codes, are summarized in Table 3. The N-terminal domain of SPT16 is highly conserved with a "pita-bread" or "baseball glove" like shape, which is characteristic of amino-peptidases, but yet, no amino-peptidase activity has been reported for the SPT16 NTD. To date, only one structure of SPT16 is available from a plant source; i.e., SPT16 NTD from Cicer arietinum (PDB id, 5CE6) (Are et al. 2016).

The Cicer arietinum SPT16 NTD shows $63.8 \%$ and $31 \%$ sequence identity with Arabidopsis and human SPT16 NTD, respectively. However, no structures are available for other domains from any plant source. The sequence comparison of AtSPT16 with human, yeast, and $C$. arietinum shows that in AtSPT16, the NTD probably covers the residue stretch 1-460 and the stretch 663-954 forms the middle domain.

The sequence alignments and the secondary structure predictions showed that almost all the $\alpha$-helices and $\beta$-strands in A. thaliana are conserved and are similar to that of C. arietinum. The structure of CaSPT16 NTD consists of two distinct structural domains; an N-terminal lobe (residue S22-A196) and a C-terminal lobe (residues S198-S456), connected by K197 forming a hinge (Fig. 6a) (Are et al. 2016). In A. thaliana, the residues S25-A199 form the N-terminal lobe and the hinge has the conserved residue K200. The structure of $C$. arietinum SPT16 NTD (CaSPT16N) (PDB id, 5CE6) and its comparison with $S$. cerevisiae SPT16 (PDB id, 3BIT) are shown in Fig. 6b. The chain B of ScSPT16 was used for the alignment and the overall r.m.s.d. for the $\mathrm{C} \alpha$ atoms was $1.94 \AA$. To compare the individual lobes and the full-length of CaSPT16 NTD with yeast and human SPT16, we made the r.m.s.d. calculations and we see considerable differences in the inter-domain angles of SPT16 NTD at the hinge region formed by the residue K197. The inter-lobe angle in CaSPT16 NTD was $108^{\circ}$. However, it was $113^{\circ}, 114^{\circ}$, and $115^{\circ}$ in $S$. pombe, $S$. cerevisiae, and $H$. sapiens, respectively. The NTD has two conserved surfaces that are negatively charged and seemingly crucial for the binding of $\mathrm{H} 3 / \mathrm{H} 4$, and it has been predicted that the open conformation of LXXS loop of CaSPT16N may provide access to a 
Yeast
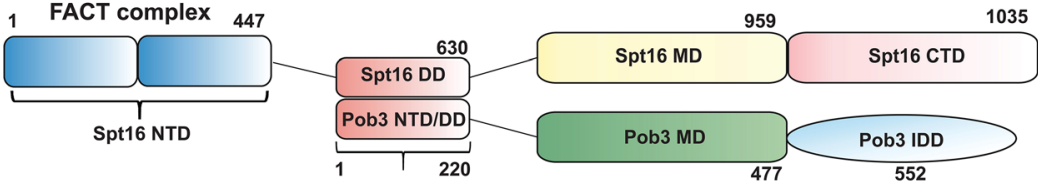

ScSpt16 (1035aa)

Spt16 NTD
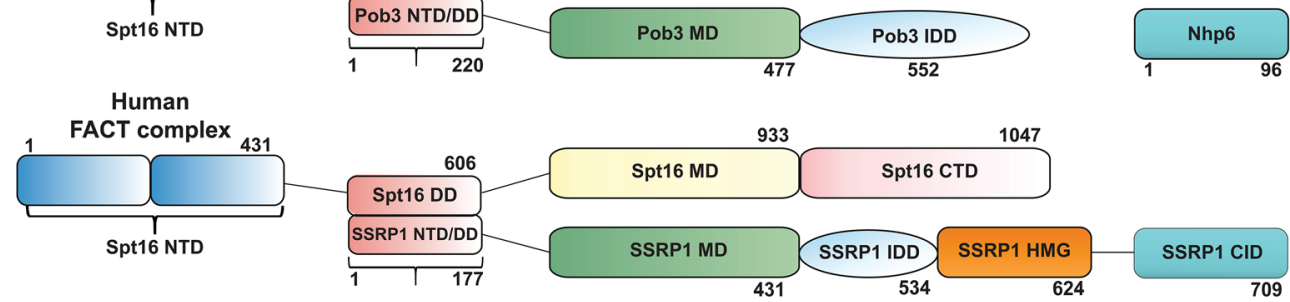

ScPob3 (552aa)
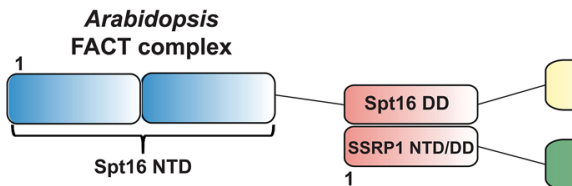

Spt16 MD

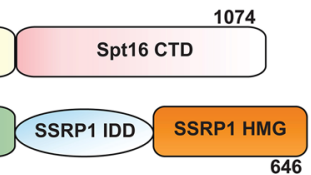

AtSpt16 (1074aa)

AtSSRP1 (646aa)

Fig. 5 The domain organization of the FACT subunits from $S$. cerevisiae, H. sapiens, and A. thaliana. NTD, N-terminal domain; DD, dimerization domain; MD, middle domain; CTD, C-terminal domain; IDD, intrinsically disordered domain; CID, C-terminal intrinsically disordered domain; HMG, high mobility group domain hydrophobic docking site on SPT16 NTD for the hydrophobic residues from histone $\mathrm{H} 3 / \mathrm{H} 4$ tails (Fig. 6a) (Are et al. 2016). SPT16 NTD is known to bind to the N-terminal tails of H3-H4 tetramers with an affinity in micrometer range (Stuwe et al. 2008; VanDemark et al. 2006).

The DD domain helps in dimerization with SSRP1 NTD/ DD, while the middle domain consists of two "Pleckstrinlike" or "Pleckstrin homology" $(\mathrm{PH})$ motifs, which are known to interact with $\mathrm{H} 2 \mathrm{~B}$ by electrostatic interactions. The $\mathrm{PH}$ motif in the middle domain facilitates proper binding to an H2A-H2B dimer, but the full-length FACT complex interacts with histone dimer with a higher affinity than the middle domain, hinting towards the presence of some additional epitopes involved in the interaction (Bondarenko et al. 2015; Hondele et al. 2013). Furthermore, AtSPT16 residues 663954 show $44 \%$ identity with the middle domain of SPT16 of $C$. thermophilum and could be similar in structure in A. thaliana SPT16 also. We further modeled the middle domain of AtSPT16 using Phyre2 server and compared it with $S$. cerevisiae and $C$. thermophilum middle domain structures. All the residues of the model got built with $100 \%$ confidence. Further, the AtSPT16 MD model shows an r.m.s.d. of only 0.5 $\AA$ with both $S$. cerevisiae and C. thermophilum structures. The smaller r.m.s.d. value shows that the structure of the middle domain of the FACT protein complex to be highly conserved across the different species. Next, a helix is absent in the region corresponding to AtSPT16 MD $\alpha 3$ in both $S$. cerevisiae and $C$. thermophilum structures. This region could become disordered and crucial in the function of the protein. All the $\beta$-sheets are remarkably conserved, and the significant differences mostly lie in the position of loop regions. CTD of SPT16 is also known to be involved in histone binding and consists of a long stretch of glutamic acid residues, providing a strong negative charge to the protein. Deletion of this domain in $S$. cerevisiae abolished the binding of H2A-H2B and turned lethal for the yeast cells (Bondarenko et al. 2015; Winkler and Luger 2011; Zhou et al. 2015). The CTD of $A$. thaliana SPT16 is also negatively charged due to glutamic acid residues, and their importance in histone interaction still needs to be assessed.

The SSRP1/Pob3 subunit is of 646-residue length in A. thaliana and is slightly longer in human (709 residues) but is smaller in yeast (552 residues). Similar to SPT16, SSRP1 subunit also consists of an NTD/DD domain that is involved in heterodimerization with SPT16 to form the FACT complex, an MD domain with two PH folds and a CTD or intrinsically disordered domain (IDD) with a high content of acidic residues that is typical in all histone chaperones. The SSRP1 could show functions independent of SPT16 as well. The human and Dictyostelium discoideum SSRP1, both from elongated homodimers using $\mathrm{PH} 2$ and $\mathrm{PH} 3$ domains, as well as they bind both $\mathrm{H} 2 \mathrm{~A}-\mathrm{H} 2 \mathrm{~B}$ and $\mathrm{H} 3-\mathrm{H} 4$ in solution (Marciano et al. 2018). The homo-dimerization is shown to be a concentration-dependent phenomenon and occurs only at higher concentrations. However, at a lower concentration, it hetero-dimerizes with SPT16 subunit (Marciano et al. 2018). Also, whether this dimerization is a universal phenomenon and how it is regulated needs to be further analyzed in details. Although the overall PH domain and IDD are conserved across the species, ranging from the plant, fungi to mammals in SSRP1, a closer inspection reveals that a conserved residue Gln306 has gotten mutated to Lys in plants. Dissecting the dimerization property of plant SSRP1 could give more clues regarding a possible independent function in the plant. Also, structural studies would be needed to evaluate the actual dimerization residues in this protein further. More recently, the 
Table 3 SPT16 and SSRP1/Pob3 structures

\begin{tabular}{|c|c|c|c|c|c|}
\hline Name & Source organism & $\begin{array}{l}\text { PDB } \\
\text { code }\end{array}$ & $\begin{array}{l}\text { Residue } \\
\text { range }\end{array}$ & Resolution & Primary citation \\
\hline SPT16 NTD & S. cerevisiae & $\begin{array}{l}3 \mathrm{BIT} \\
3 \mathrm{BIQ}\end{array}$ & $\begin{array}{l}1-451 \\
1-465\end{array}$ & $\begin{array}{l}1.91 \AA \\
1.73 \AA\end{array}$ & $\begin{array}{l}\text { (VanDemark et al. } \\
\text { 2006) } \\
\text { (VanDemark et al. } \\
\text { 2006) }\end{array}$ \\
\hline SPT16 NTD & S. pombe & $\begin{array}{l}3 \mathrm{CB} 5 \\
3 \mathrm{CB} 6\end{array}$ & $\begin{array}{l}1-442 \\
1-442\end{array}$ & $\begin{array}{l}2.05 \AA \\
1.84 \AA\end{array}$ & $\begin{array}{l}\text { (Stuwe et al. 2008) } \\
\text { (Stuwe et al. 2008) }\end{array}$ \\
\hline SPT16 NTD & C. arietinum & 5 CE6 & $1-480$ & $1.7 \AA$ & (Are et al. 2016) \\
\hline SPT16 NTD & H. sapiens & $\begin{array}{l}5 \mathrm{E} 5 \mathrm{~B} \\
5 \mathrm{UMT} \\
5 \mathrm{XM} 2\end{array}$ & $\begin{array}{l}1-510 \\
1-434 \\
1-437\end{array}$ & $\begin{array}{l}1.8 \AA \\
2.09 \AA \\
2.18 \AA\end{array}$ & $\begin{array}{l}\text { (Marciano and } \\
\text { Huang 2016) } \\
\text { (Unpublished) } \\
\text { (Unpublished) }\end{array}$ \\
\hline SPT16 NTD & A. gossypii & $6 \mathrm{~A} 8 \mathrm{M}$ & $1-461$ & $1.7 \AA$ & (Unpublished) \\
\hline SPT16 MD & S. cerevisiae & $4 \mathrm{IOY}$ & $675-958$ & $1.95 \AA$ & $\begin{array}{l}\text { (Kemble et al. } \\
\text { 2013) }\end{array}$ \\
\hline SPT16 MD & C. thermophilum & $4 \mathrm{KHO}$ & $651-945$ & $2.0 \AA$ & $\begin{array}{l}\text { (Hondele et al. } \\
\text { 2013) }\end{array}$ \\
\hline SPT16 MD & H. sapiens & $4 \mathrm{Z} 2 \mathrm{~N}$ & $644-930$ & $1.92 \AA$ & $\begin{array}{l}\text { (Tsunaka et al. } \\
\text { 2016) }\end{array}$ \\
\hline SSRP1 HMGB domain & $\begin{array}{l}\text { D. melanogaster } \\
\text { H. sapiens }\end{array}$ & $\begin{array}{l}1 \mathrm{WXL} \\
5 \mathrm{VWE}\end{array}$ & $\begin{array}{l}555-624 \\
551-617\end{array}$ & $\begin{array}{l}\text { By NMR } \\
\text { By NMR }\end{array}$ & $\begin{array}{l}\text { (Kasai et al. 2005) } \\
\text { (Unpublished) }\end{array}$ \\
\hline $\begin{array}{l}\text { Middle double PH } \\
\text { domain of SPT16 }\end{array}$ & H. sapiens & $5 \mathrm{UMU}$ & $649-926$ & $1.903 \AA$ & (Unpublished) \\
\hline SSRP1 NTD & H. sapiens & 5UMR & $1-102$ & $1.5 \AA$ & (Unpublished) \\
\hline $\begin{array}{l}\text { Middle double PH } \\
\text { domain of SSRP1 }\end{array}$ & H. sapiens & 5UMS & $193-430$ & $1.56 \AA$ & (Unpublished) \\
\hline SSRP1 MD & H. sapiens & 4IFS & $196-430$ & $1.93 \AA$ & (Unpublished) \\
\hline SPT16: Pob3 heterodimer & C. thermophilum & $4 \mathrm{KHB}$ & $\begin{array}{l}522-647 \\
\quad \text { (SPT16) } \\
1-192 \\
\text { (Pob3) }\end{array}$ & $2.4 \AA$ & $\begin{array}{l}\text { (Hondele et al. } \\
\text { 2013) }\end{array}$ \\
\hline SPT16:H2A-H2B & S. cerevisiae & $4 \mathrm{WNN}$ & $\begin{array}{c}958-990 \\
\text { (SPT16) } \\
16-101 \\
(\mathrm{H} 2 \mathrm{~A}) \\
29-126 \\
(\mathrm{H} 2 \mathrm{~B})\end{array}$ & $1.8 \AA$ & $\begin{array}{l}\text { (Kemble et al. } \\
\text { 2015) }\end{array}$ \\
\hline SPT16 MD:H3-H4 & H. sapiens & $4 \mathrm{Z} 2 \mathrm{M}$ & $\begin{array}{c}\text { 644-930 } \\
\text { (SPT16) } \\
35-136 \\
(\mathrm{H} 3.1) \\
23-93(\mathrm{H} 4)\end{array}$ & $2.98 \AA$ & $\begin{array}{l}\text { (Tsunaka et al. } \\
\text { 2016) }\end{array}$ \\
\hline
\end{tabular}

NTD N-terminal domain, $M D$ middle domain, $P H$ pleckstrin homology domain role of A. thaliana SSRP1 has been shown in seed dormancy where it is identified as a novel factor that modulates seed dormancy through the efficient expression of DOG1 (MichlHolzinger et al. 2019).

The organization of SSRP1 in A. thaliana seems similar to human SSRP1. The sequence alignment of HsSSRP1, ScPob3, and AtSSRP1 shows $12 \%$ of the residues to be utterly conserved among the three. Upon closer observation in comparison with HsSSRP1, we found the changes getting localized as patches distributed all along, but the $\mathrm{C}$-terminus part, which probably constitutes the HMG domain has a higher concentration of conserved residues. The structure appears to be primarily made up of $\beta$-strands, forming antiparallel $\beta$-sheets, which is characteristic of most of the histonebinding chaperones. The extended loop region (V328-G334) connecting the two lobes of HsSSRP1 protein, is relatively well conserved in AtSSRP1 as well. The human and A. thaliana SSRP1 consists of an additional domain called 
a

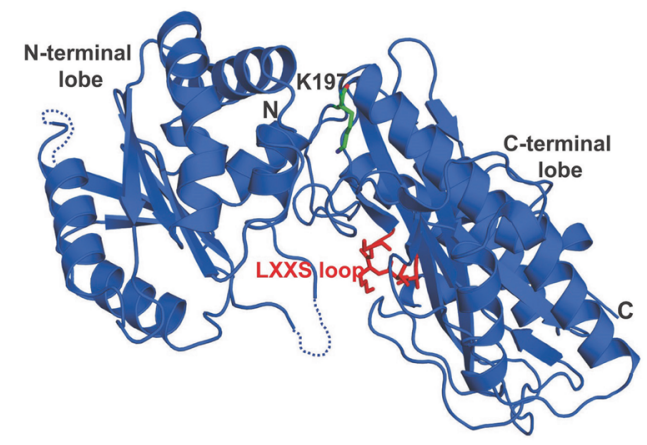

b

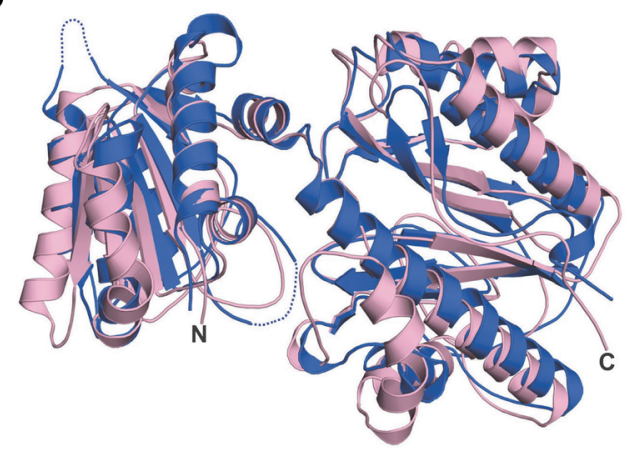

C

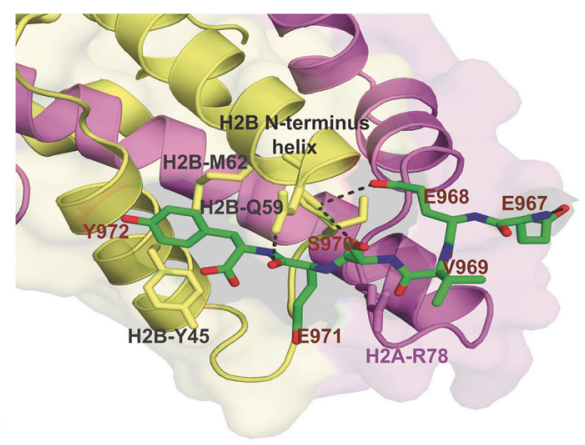

d

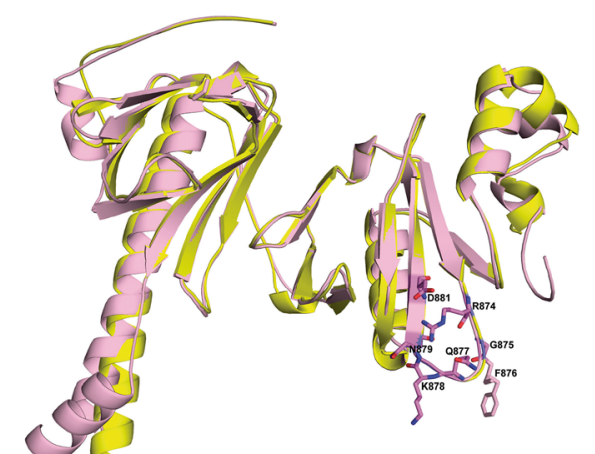

e
Fig. 6 FACT domain structures. a The structure of $C$. arietinum SPT16 NTD (PDB id, 5CE6). The important structural features are labeled. The K197 residue, which provides a hinge and separates the two domains, is represented in stick model in green and the LXXS loop in stick model and red. b The structural overlap of SPT16 NTDs from $C$. arietinum and $S$. cerevisiae (PDB id, 3BIT). C. arietinum SPT16 NTD is in blue and $S$. cerevisiae SPT16 NTD is in light pink. The r.m.s.d. of $\mathrm{C} \alpha$ atoms obtained after alignment was $1.94 \AA$. c The close-up view of histone interaction from the structure of $S$. cerevisiae SPT16-H2A-H2B complex (PDB id, 4WNN). H2B is in yellow, and $\mathrm{H} 2 \mathrm{~A}$ is in purple. The residues of H2B forming hydrophobic pocket are shown in stick representation and are labeled. The SPT16 NTD peptide is in stick representation in green, and all the residues are marked. $\mathbf{d}$ The structure overlap of AtSPT16 middle domain (pink) and HsSPT16 MD (yellow) (PDB id, 4Z2N). The r.m.s.d. of $\mathrm{C} \alpha$ atoms obtained after alignment was $1.94 \AA$. The conserved histone interacting residues in AtSPT16 are shown in stick model. e The structure of $D$. melanogaster SSRP1- HMG box domain (PDB id, 1WXL). The three helices that form the L-shaped fold, typical of HMGB domain are labeled
HMG domain, but the yeast Pob3 lacks this domain and instead contains a small domain of 93 residue length, named Nhp6 (Bondarenko et al. 2015; Bruhn et al. 1992; Formosa et al. 2001; Kasai et al. 2005; Zhou et al. 2015). The HMGB domain binds to a minor groove of DNA and without any sequence specificity (Thomas and Stott 2012). This DNAbending property of HMGB helps in destabilizing nucleosome by disrupting the electrostatic contacts between the DNA and histones (McCullough et al. 2018). Furthermore, the DNAbending property of HMGB promotes nucleosome assembly by exposing FACT's histone-binding sites, and the excessive activity serves as a quality control check-point during nucleosome assembly (McCullough et al. 2018). An NMR structure of Drosophila SSRP1 HMGB domain (PDB id, 1WXL) is available, and the structure reveals three $\alpha$-helices, which are connected by loops, forming an L-shaped fold, typical for HMGB domains (Fig. 6d) (Kasai et al. 2005). Similarly, A. thaliana SSRP1 (UniProtKB id, Q05153) also contains an HMGB domain, and for this, the DNA binding region appears to lie between the residues 561-630. The plant SSRP1 binds preferably to distorted DNA and also has a DNA-bending activity due to the HMGB domain. However, there is no sequence specificity for this DNA interaction in plants as well (Pfab et al. 2018a). The HMG box domain is not critical for the survival of the plant, although the FACT complex in HMGB mutant lines lose their ability to bend DNA and its interaction with nucleosome (Pfab et al. 2018b).

\section{FACT-histone complex structures}

A study on FACT in complex with $\mathrm{H} 2 \mathrm{~A}-\mathrm{H} 2 \mathrm{~B}$ has shown that $\mathrm{H} 2 \mathrm{~A}-\mathrm{H} 2 \mathrm{~B}$ binding is a core activity of this chaperone and this interaction gets mediated by the natively unstructured domain present in the C-terminal tail of SPT16 and Pob3 heterodimer (Kemble et al. 2015). The structural studies have revealed that this interaction occurs in 1:1 ratio of FACT and $\mathrm{H} 2 \mathrm{~A}-\mathrm{H} 2 \mathrm{~B}$ and the C-terminal region of both SPT16 and Pob3 competes for the same binding site on $\mathrm{H} 2 \mathrm{~A}-\mathrm{H} 2 \mathrm{~B}$, and both can bind to one 
copy of the H2A-H2B dimer. This study provides a model for the mechanism of nucleosomal reorganization by FACT complex as it competes with DNA for its H2A-H2B binding and thus impedes the second and the thirteenth binding site of DNA on H2A-H2B. In a previous study, the crystal structure of $C$. thermophilum SPT16M in complex with H2A-H2B has been reported (Hondele et al. 2013); however, there appears to be some ambiguity in that structure due to the artifacts that have come about due to the construct design (Kemble et al. 2015).

Nucleosome reorganization mechanism by human FACT complex has also been studied (Tsunaka et al. 2016). A double-strand break in the vicinity of H2B-N-terminal tail facilitates FACT to make a complex with the nucleosome. The association of FACT with nucleosome helps FACT middomain to interact with $\mathrm{H} 2 \mathrm{~A}$-interacting face of $(\mathrm{H} 3-\mathrm{H} 4)_{2}$ followed by displacement of H2A-H2B from the nucleosome, which ultimately leads to the nucleosomal DNA ends from the histone octamer. This crystal structure of hFACT MD-(H3$\mathrm{H} 4)_{2}$ has helped in understanding how the middle domain and the highly acidic segment (AID) interact with histones within the nucleosome. The crystal structure reveals an unconventional binding of histone tetramer in contrast to histone dimer binding to other histone chaperones like ASF1 (English et al. 2006; Natsume et al. 2007). There are two binding sites for a histone tetramer. The first binding site is constituted of antiparallel $\beta$-sheets and a $\beta$-strand of hMIDAID of the FACT complex. This binding site does not show any sequence identity with any of the known histone chaperones which also binds $\mathrm{H} 3-\mathrm{H} 4$ tetramer, though it shows as a structural similarity. The second binding site contains evolutionarily conserved residues across species from yeast to humans. Six residues (Arg847, Gln849, Leu852, Lys853, Asn854, and Asp856) form the histone-binding loop at the second site of mid-domain of human FACT complex (Tsunaka et al. 2016). These residues are conserved in A. thaliana SPT16 MD as well. The conserved residues have been highlighted in Fig. 6e. Thus, the binding mechanism of the mid-domain could be similar in Arabidopsis as well. The binding of a single copy of H2A-H2B dimer with human FACT has been reported, allowing the simultaneous binding of a histone H3-H4 tetramer (Wang et al. 2018). The binding of histone dimer facilitates the proper interaction of tetramer in the ternary complex. This work proposes a model of how FACT and histones together form an intermediate during nucleosome formation.

The domain structures of all subunits in human FACT complex are available in PDB (Table 3). However, due to the dynamic functions of FACT, structural studies on plant FACT-histone complexes is also warranted to understand the dynamic nature of plant gene regulation. Also, the structural studies on post-translationally modified FACT with histones and even nucleosomes would be significant to understand the stability of plant nucleosomes. Single-particle cryo-electron microscopy studies on plant FACT in complex with nucleosome could be a challenging task but could provide a wealth of information about the chromatin dynamics in plants.

\section{Conclusion}

The histone chaperones form a relatively extensive repertoire in the eukaryotic nucleus and are structurally distinct from each other. They function crucially in almost all aspects of chromatin biology, starting from histone transport to nucleosome assembly and disassembly. The plants must respond quickly against any stress conditions like heat, cold, drought, and pathogen infections, and they have evolved themselves with sufficient machinery to react against these conditions. Significant progress has been achieved in understanding the transcriptional regulation and epigenetic changes during the quick responses in plants against stress conditions. When it comes to the plant histone chaperones, we have a long way to go in understanding the histone chaperones and their complexes that play definitive roles in chromatin organization and dynamics. Though putative sequences of several families of histone chaperones in plants are known, no significant effort has gone to study them structurally or functionally, other than what has been discussed in this review. Structural studies on plant histone chaperones and their interacting partners need to see a revived interest. Gene regulation mechanism in plants is quite dynamic and structural studies on plant histone chaperones will be of great value not only from the plant gene regulation point of view but also for a better understanding about the evolution of these histone chaperones and how the complex organisms have evolved their genetic machinery to survive various adverse conditions. A recent review gives an excellent summary of the role of histone variants in plant abiotic stress conditions (Liu et al. 2015). Studies should look at the role of regulation of transcription machinery in plants by histone chaperones, especially during different biotic and abiotic stress conditions. The histone chaperones specific for the different variant histones can be another prospective area for research that would improve our understanding of plant chromatin dynamics. Also, the structural studies of complexes with full-length histone chaperones and histones have to be addressed in the future to gain more mechanistic insights on the function of these proteins. To date, no structures are available for any histone chaperones in complex with the nucleosome. Though challenging, it would help to understand chromatin dynamics through the structural analyses of such complexes. Another critical question to be addressed is to find the common evolutionary characteristics among all known histone chaperones and to understand how they control the exchange of histones in vivo. The presence of intrinsically disordered regions (IDRs) and highly charged acidic stretches 
form a standard feature of the different histone chaperone families. 23 out of 28 human histone chaperones contain such disordered regions (Warren and Shechter 2017). These IDRs appear highly plastic, providing a high degree of conformational flexibility, allowing them to interact with multiple ligands and also allowing it to fine-tune its protein-protein interaction network. The flexibility also allows them to undergo a variety of post-translational modifications and thus a charge shift in them, probably forming a switching mechanism. In NPM2, phosphorylation in IDR leads to increased binding affinity to histones (Onikubo et al. 2015; Warren and Shechter 2017). Studying the dynamics of these chaperones with extensive IDRs will be a challenge. However, recent advancements in the techniques like NMR, Cryo-EM and integrative structural biology could contribute positively to address questions on these chaperones with disordered regions, and the kind of structural changes brought about in them when they interact with the histones and chromatin.

Acknowledgements The authors acknowledge the intramural support from the Institute of Life Sciences, Bhubaneswar, and the research fellowship to A.K. from the Department of Biotechnology, Government of India.

\section{Compliance with ethical standards}

Conflict of interest The authors declare that they have no conflict of interest.

\section{References}

Aguilar-Gurrieri C, Larabi A, Vinayachandran V, Patel NA, Yen K, Reja R, Ebong IO, Schoehn G, Robinson CV, Pugh BF, Panne D (2016) Structural evidence for Nap1-dependent H2A-H2B deposition and nucleosome assembly. EMBO J 35:1465-1482

Are VN, Ghosh B, Kumar A, Gadre R, Makde RD (2016) Crystal structure and dynamics of Spt16N-domain of FACT complex from Cicer arietinum. Int J Biol Macromol 88:36-43

Avvakumov N, Nourani A, Cote J (2011) Histone chaperones: modulators of chromatin marks. Mol Cell 41:502-514

Barna B, Gémes K, Domoki M, Bernula D, Ferenc G, Bálint B, Nagy I, Fehér A (2018) Arabidopsis NAP-related proteins (NRPs) contribute to the coordination of plant growth, developmental rate, and agerelated pathogen resistance under short days. Plant Sci 267:124-134

Berndsen CE et al (2008) Molecular functions of the histone acetyltransferase chaperone complex Rtt109-Vps75. Nat Struct Mol Biol 15: 948-956

Bondarenko MT, Maluchenko NV, Valieva ME, Gerasimova NS, Kulaeva OI, Georgiev PG, Studitsky VM (2015) Structure and function of histone chaperone FACT. Mol Biol (Mosk) 49:891-904

Bourque $\mathrm{S}$ et al (2016) The evolution of HD2 proteins in green plants trends. Plant Sci 21:1008-1016

Bruhn SL, Pil PM, Essigmann JM, Housman DE, Lippard SJ (1992) Isolation and characterization of human cDNA clones encoding a high mobility group box protein that recognizes structural distortions to DNA caused by binding of the anticancer agent cisplatin. Proc Natl Acad Sci U S A 89:2307-2311
Burglin TR, De Robertis EM (1987) The nuclear migration signal of Xenopus laevis nucleoplasmin. EMBO J 6:2617-2625

Chen H, Li B, Workman JL (1994) A histone-binding protein, nucleoplasmin, stimulates transcription factor binding to nucleosomes and factor-induced nucleosome disassembly. EMBO J 13: 380-390

Cotten M, Sealy L, Chalkley R (1986) Massive phosphorylation distinguishes Xenopus laevis nucleoplasmin isolated from oocytes or unfertilized eggs. Biochemistry 25:5063-5069

D'Arcy S et al (2013) Chaperone Nap1 shields histone surfaces used in a nucleosome and can put $\mathrm{H} 2 \mathrm{~A}-\mathrm{H} 2 \mathrm{~B}$ in an unconventional tetrameric form. Mol Cell 51:662-677

Dong A, Zhu Y, Yu Y, Cao K, Sun C, Shen WH (2003) Regulation of biosynthesis and intracellular localization of rice and tobacco homologues of nucleosome assembly protein 1. Planta 216:561-570

Drane P, Ouararhni K, Depaux A, Shuaib M, Hamiche A (2010) The death-associated protein DAXX is a novel histone chaperone involved in the replication-independent deposition of H3.3. Genes Dev 24:1253-1265

Duroux M, Houben A, Ruzicka K, Friml J, Grasser KD (2004) The chromatin remodelling complex FACT associates with actively transcribed regions of the Arabidopsis genome. Plant J 40:660-671

Dutta S, Akey IV, Dingwall C, Hartman KL, Laue T, Nolte RT, Head JF, Akey CW (2001) The crystal structure of nucleoplasmin-core: implications for histone binding and nucleosome assembly. Mol Cell 8: 841-853

Earnshaw WC, Honda BM, Laskey RA, Thomas JO (1980) Assembly of nucleosomes: the reaction involving X. laevis nucleoplasmin. Cell 21:373-383

Edlich-Muth C, Artero JB, Callow P, Przewloka MR, Watson AA, Zhang W, Glover DM, Debski J, Dadlez M, Round AR, Forsyth VT, Laue ED (2015) The pentameric nucleoplasmin fold is present in Drosophila FKBP39 and a large number of chromatin-related proteins. J Mol Biol 427:1949-1963

Elsaesser SJ, Allis CD (2010) HIRA and Daxx constitute two independent histone H3.3-containing predeposition complexes. Cold Spring Harb Symp Quant Biol 75:27-34

Elsasser SJ (2013) A common structural theme in histone chaperones mimics interhistone contacts. Trends Biochem Sci 38:333-336

Elsasser SJ, Huang H, Lewis PW, Chin JW, Allis CD, Patel DJ (2012) DAXX envelops a histone H3.3-H4 dimer for H3.3-specific recognition. Nature 491:560-565

English CM, Adkins MW, Carson JJ, Churchill ME, Tyler JK (2006) Structural basis for the histone chaperone activity of Asfl. Cell 127:495-508

Formosa T, Eriksson P, Wittmeyer J, Ginn J, Yu Y, Stillman DJ (2001) Spt16-Pob3 and the HMG protein Nhp6 combine to form the nucleosome-binding factor SPN. EMBO J 20:3506-3517

Franco A et al (2019) Structural insights into the ability of nucleoplasmin to assemble and chaperone histone octamers for DNA deposition. Sci Rep 9:9487

Frehlick LJ, Eirin-Lopez JM, Jeffery ED, Hunt DF, Ausio J (2006) The characterization of amphibian nucleoplasmins yields new insight into their role in sperm chromatin remodeling. BMC Genomics 7:99

Gill J et al (2009) Crystal structure of malaria parasite nucleosome assembly protein: distinct modes of protein localization and histone recognition. J Biol Chem 284:10076-10087

Gill J et al (2010) Structure, localization and histone binding properties of nuclear-associated nucleosome assembly protein from Plasmodium falciparum. Malar J 9:90

Hammond CM et al (2016) The histone chaperone Vps75 forms multiple oligomeric assemblies capable of mediating exchange between histone H3-H4 tetramers and Asf1-H3-H4 complexes. Nucleic Acids Res 44:6157-6172

Hansen DF, Zhou Z, Feng H, Miller Jenkins LM, Bai Y, Kay LE (2009) Binding kinetics of histone chaperone Chz1 and variant histone 
H2A.Z-H2B by relaxation dispersion NMR spectroscopy. J Mol Biol 387:1-9

Hondele M, Stuwe T, Hassler M, Halbach F, Bowman A, Zhang ET, Nijmeijer B, Kotthoff C, Rybin V, Amlacher S, Hurt E, Ladurner AG (2013) Structural basis of histone H2A-H2B recognition by the essential chaperone FACT. Nature 499:111-114

Hsieh FK, Kulaeva OI, Patel SS, Dyer PN, Luger K, Reinberg D, Studitsky VM (2013) Histone chaperone FACT action during transcription through chromatin by RNA polymerase II. Proc Natl Acad Sci U S A 110:7654-7659

Ishimi Y, Kikuchi A (1991) Identification and molecular cloning of yeast homolog of nucleosome assembly protein I which facilitates nucleosome assembly in vitro. J Biol Chem 266:7025-7029

Ito T, Bulger M, Kobayashi R, Kadonaga JT (1996) Drosophila NAP-1 is a core histone chaperone that functions in ATP-facilitated assembly of regularly spaced nucleosomal arrays. Mol Cell Biol 16:31123124

Kasai N, Tsunaka Y, Ohki I, Hirose S, Morikawa K, Tate S (2005) Solution structure of the HMG-box domain in the SSRP1 subunit of FACT. J Biomol NMR 32:83-88

Kelley LA, Mezulis S, Yates CM, Wass MN, Sternberg MJ (2015) The Phyre2 web portal for protein modeling, prediction and analysis. Nat Protoc 10:845-858

Kemble DJ, Whitby FG, Robinson H, McCullough LL, Formosa T, Hill CP (2013) Structure of the Spt16 middle domain reveals functional features of the histone chaperone FACT. J Biol Chem 288:1018810194

Kemble DJ, McCullough LL, Whitby FG, Formosa T, Hill CP (2015) FACT Disrupts Nucleosome Structure by Binding H2A-H2B with Conserved Peptide Motifs. Mol Cell 60:294-306

Kozlowska M et al (2017) Nucleoplasmin-like domain of FKBP39 from Drosophila melanogaster forms a tetramer with partly disordered tentacle-like C-terminal segments. Sci Rep 7:40405

Kumar A, Kumar Singh A, Chandrakant Bobde R, Vasudevan D (2019) Structural characterization of Arabidopsis thaliana NAP1-related protein 2 (AtNRP2) and comparison with its homolog AtNRP1 molecules 24

Laskey RA, Honda BM, Mills AD, Finch JT (1978) Nucleosomes are assembled by an acidic protein which binds histones and transfers them to DNA. Nature 275:416-420

Le S, Davis C, Konopka JB, Sternglanz R (1997) Two new S-phasespecific genes from Saccharomyces cerevisiae. Yeast 13:1029-1042

Li F et al (2016) Structural insights into the interaction and disease mechanism of neurodegenerative disease-associated optineurin and TBK1 proteins. Nat Commun 7:12708

Liu Z, Zhu Y, Gao J, Yu F, Dong A, Shen WH (2009) Molecular and reverse genetic characterization of nucleosome assembly protein 1 (NAP1) genes unravels their function in transcription and nucleotide excision repair in Arabidopsis thaliana. Plant J 59:27-38

Liu J, Feng L, Li J, He Z (2015) Genetic and epigenetic control of plant heat responses. Front Plant Sci 6:267

Lolas IB et al (2010) The transcript elongation factor FACT affects Arabidopsis vegetative and reproductive development and genetically interacts with HUB1/2. Plant J 61:686-697

Lovell SC et al (2003) Structure validation by Calpha geometry: phi,psi and Cbeta deviation. Proteins 50:437-450

Luger K, Mader AW, Richmond RK, Sargent DF, Richmond TJ (1997) Crystal structure of the nucleosome core particle at 2.8 A resolution. Nature 389:251-260

Maksimov V, Nakamura M, Wildhaber T, Nanni P, Ramstrom M, Bergquist J, Hennig L (2016) The H3 chaperone function of NASP is conserved in Arabidopsis. Plant J 88:425-436

Marciano G, Huang DT (2016) Structure of the human histone chaperone FACT Spt16 N-terminal domain. Acta Crystallogr F Struct Biol Commun 72:121-128
Marciano G, Da Vela S, Tria G, Svergun DI, Byron O, Huang DT (2018) Structure-specific recognition protein-1 (SSRP1) is an elongated homodimer that binds histones. J Biol Chem 293:10071-10083

McCullough LL, Connell Z, Xin H, Studitsky VM, Feofanov AV, Valieva ME, Formosa T (2018) Functional roles of the DNA-binding HMGB domain in the histone chaperone FACT in nucleosome reorganization. J Biol Chem 293:6121-6133

Michl-Holzinger P, Mortensen SA, Grasser KD (2019) The SSRP1 subunit of the histone chaperone FACT is required for seed dormancy in Arabidopsis. J Plant Physiol 236:105-108

Mitrea DM et al (2014) Structural polymorphism in the N-terminal oligomerization domain of NPM1. Proc Natl Acad Sci U S A 111:44664471

Muto S, Senda M, Akai Y, Sato L, Suzuki T, Nagai R, Senda T, Horikoshi M (2007) Relationship between the structure of SET/TAF-Ibeta/ INHAT and its histone chaperone activity. Proc Natl Acad Sci U S A 104:4285-4290

Namboodiri VM, Dutta S, Akey IV, Head JF, Akey CW (2003) The crystal structure of Drosophila NLP-core provides insight into pentamer formation and histone binding. Structure 11:175-186

Namboodiri VM, Akey IV, Schmidt-Zachmann MS, Head JF, Akey CW (2004) The structure and function of Xenopus NO38-core, a histone chaperone in the nucleolus. Structure 12:2149-2160

Natsume R, Eitoku M, Akai Y, Sano N, Horikoshi M, Senda T (2007) Structure and function of the histone chaperone CIA/ASF1 complexed with histones H3 and H4. Nature 446:338-341

Obri A, Ouararhni K, Papin C, Diebold ML, Padmanabhan K, Marek M, Stoll I, Roy L, Reilly PT, Mak TW, Dimitrov S, Romier C, Hamiche A (2014) ANP32E is a histone chaperone that removes H2A.Z from chromatin. Nature 505:648-653

Oliveira DV et al (2014) Histone chaperone FACT regulates homologous recombination by chromatin remodeling through interaction with RNF20. J Cell Sci 127:763-772

Onikubo T et al (2015) Developmentally regulated post-translational modification of nucleoplasmin controls histone sequestration and deposition. Cell Rep 10:1735-1748

Orphanides G, Wu WH, Lane WS, Hampsey M, Reinberg D (1999) The chromatin-specific transcription elongation factor FACT comprises human SPT16 and SSRP1 proteins. Nature 400:284-288

Padeken J, Mendiburo MJ, Chlamydas S, Schwarz HJ, Kremmer E, Heun P (2013) The nucleoplasmin homolog NLP mediates centromere clustering and anchoring to the nucleolus. Mol Cell 50:236-249

Park YJ, Luger K (2006a) Structure and function of nucleosome assembly proteins. Biochem Cell Biol 84:549-558

Park YJ, Luger K (2006b) The structure of nucleosome assembly protein 1. Proc Natl Acad Sci U S A 103:1248-1253

Park YJ, Luger K (2008) Histone chaperones in nucleosome eviction and histone exchange. Curr Opin Struct Biol 18:282-289

Park YJ, McBryant SJ, Luger K (2008) A beta-hairpin comprising the nuclear localization sequence sustains the self-associated states of nucleosome assembly protein 1. J Mol Biol 375:1076-1085

Pfab A, Breindl M, Grasser KD (2018a) The Arabidopsis histone chaperone FACT is required for stress-induced expression of anthocyanin biosynthetic genes. Plant Mol Biol 96:367-374

Pfab A, Gronlund JT, Holzinger P, Langst G, Grasser KD (2018b) The Arabidopsis histone chaperone FACT: role of the HMG-box domain of SSRP1. J Mol Biol 430:2747-2759

Philpott A, Leno GH, Laskey RA (1991) Sperm decondensation in Xenopus egg cytoplasm is mediated by nucleoplasmin. Cell 65 : 569-578

Platonova O, Akey IV, Head JF, Akey CW (2011) Crystal structure and function of human nucleoplasmin (npm2): a histone chaperone in oocytes and embryos. Biochemistry 50:8078-8089

Ramos I, Martín-Benito J, Finn R, Bretaña L, Aloria K, Arizmendi JM, Ausió J, Muga A, Valpuesta JM, Prado A (2010) Nucleoplasmin 
binds histone H2A-H2B dimers through its distal face. J Biol Chem 285:33771-33778

Ray A, Lindahl E, Wallner B (2012) Improved model quality assessment using ProQ2. BMC Bioinformatics 13:224

Reinberg D, Sims RJ 3rd (2006) de FACTo nucleosome dynamics. J Biol Chem 281:23297-23301

Rottgers K, Krohn NM, Lichota J, Stemmer C, Merkle T, Grasser KD (2000) DNA-interactions and nuclear localisation of the chromosomal HMG domain protein SSRP1 from maize. Plant J 23:395-405

Sarkar P, Zhang N, Bhattacharyya S, Salvador K, D'Arcy S (2019) Characterization of Caenorhabditis elegans nucleosome assembly protein 1 uncovers the role of acidic tails in histone binding. Biochemistry 58:108-113

Smith S, Stillman B (1989) Purification and characterization of CAF-I, a human cell factor required for chromatin assembly during DNA replication in vitro. Cell 58:15-25

Spiker S (1985) Plant chromatin structure. Ann Rev Plant Physio 36:235253

Steer WM et al (2003) Xenopus nucleosome assembly protein becomes tissue-restricted during development and can alter the expression of specific genes. Mech Dev 120:1045-1057

Stuwe T, Hothorn M, Lejeune E, Rybin V, Bortfeld M, Scheffzek K, Ladurner AG (2008) The FACT Spt16 "peptidase" domain is a histone H3-H4 binding module Proc Natl Acad Sci U S A

Su D, Hu Q, Zhou H, Thompson JR, Xu RM, Zhang Z, Mer G (2011) Structure and histone binding properties of the Vps75-Rtt109 chaperone-lysine acetyltransferase complex J Biol Chem

Sugawa H, Imamoto N, Wataya-Kaneda M, Uchida T (1985) Foreign protein can be carried into the nucleus of mammalian cell by conjugation with nucleoplasmin. Exp Cell Res 159:419-429

Taneva SG et al (2008) Activation of nucleoplasmin, an oligomeric histone chaperone, challenges its stability. Biochemistry 47:1389713906

Tang Y et al (2008) Fungal Rtt109 histone acetyltransferase is an unexpected structural homolog of metazoan p300/CBP. Nat Struct Mol Biol 15:738-745

Tang Y et al (2011) Structure of the Rtt109-AcCoA/Vps75 complex and implications for chaperone-mediated histone acetylation. Structure 19:221-231

Thomas JO, Stott K (2012) H1 and HMGB1: modulators of chromatin structure. Biochem Soc Trans 40:341-346

Tripathi AK, Singh K, Pareek A, Singla-Pareek SL (2015) Histone chaperones in Arabidopsis and rice: genome-wide identification, phylogeny, architecture and transcriptional regulation. BMC Plant Biol 15: 42

Tsunaka Y, Fujiwara Y, Oyama T, Hirose S, Morikawa K (2016) Integrated molecular mechanism directing nucleosome reorganization by human FACT. Genes Dev 30:673-686

VanDemark AP, Blanksma M, Ferris E, Heroux A, Hill CP, Formosa T (2006) The structure of the yFACT Pob3-M domain, its interaction with the DNA replication factor RPA, and a potential role in nucleosome deposition. Mol Cell 22:363-374
Wang T, Liu Y, Edwards G, Krzizike D, Scherman H, Luger K (2018) The histone chaperone FACT modulates nucleosome structure by tethering its components. Life Sci Alliance 1:e201800107

Warren C, Shechter D (2017) Fly fishing for histones: catch and release by histone chaperone intrinsically disordered regions and acidic stretches. J Mol Biol 429:2401-2426

Warren C et al (2017) Dynamic intramolecular regulation of the histone chaperone nucleoplasmin controls histone binding and release. Nat Commun 8:2215

Winkler DD, Luger K (2011) The histone chaperone FACT: structural insights and mechanisms for nucleosome reorganization. J Biol Chem 286:18369-18374

Wu WH et al (2005) Swc2 is a widely conserved H2AZ-binding module essential for ATP-dependent histone exchange. Nat Struct Mol Biol 12:1064-1071

Yang J et al (2016) The histone chaperone FACT contributes to DNA replication-coupled nucleosome assembly. Cell Rep 14:1128-1141

Yogavel M, Gill J, Sharma A (2009) Iodide-SAD, SIR and SIRAS phasing for structure solution of a nucleosome assembly protein. Acta Crystallogr D Biol Crystallogr 65:618-622

Yoon HW, Kim MC, Lee SY, Hwang I, Bahk JD, Hong JC, Ishimi Y, Cho MJ (1995) Molecular cloning and functional characterization of a cDNA encoding nucleosome assembly protein 1 (NAP-1) from soybean. Mol Gen Genet 249:465-473

Zhang $M$ et al (2016) Structural insights into the association of Hifl with histones H2A-H2B dimer and H3-H4 tetramer. Structure 24:1810 1820

Zhou Z et al (2008) NMR structure of chaperone Chz1 complexed with histones H2A.Z-H2B. Nat Struct Mol Biol 15:868-869

Zhou W, Zhu Y, Dong A, Shen WH (2015) Histone H2A/H2B chaperones: from molecules to chromatin-based functions in plant growth and development. Plant J 83:78-95

Zhou W, Gao J, Ma J, Cao L, Zhang C, Zhu Y, Dong A, Shen WH (2016) Distinct roles of the histone chaperones NAP1 and NRP and the chromatin-remodeling factor INO80 in somatic homologous recombination in Arabidopsis thaliana. Plant J 88:397-410

Zhu Y, Dong A, Meyer D, Pichon O, Renou JP, Cao K, Shen WH (2006) Arabidopsis NRP1 and NRP2 encode histone chaperones and are required for maintaining postembryonic root growth. Plant Cell 18: 2879-2892

Zhu Y et al (2017) The histone chaperone NRP1 interacts with WEREWOLF to activate GLABRA2 in Arabidopsis root hair development. Plant Cell 29:260-276

Zlatanova J, Seebart C, Tomschik M (2007) Nap1: taking a closer look at a juggler protein of extraordinary skills. FASEB J 21:1294-1310

Publisher's note Springer Nature remains neutral with regard to jurisdictional claims in published maps and institutional affiliations. 